\title{
Congruences between modular forms given by the divided $\beta$ family in homotopy theory
}

\author{
MARK BEHRENS
}

\begin{abstract}
We characterize the 2-line of the $p$-local Adams-Novikov spectral sequence in terms of modular forms satisfying a certain explicit congruence condition for primes $p \geq 5$. We give a similar characterization of the 1 -line, reinterpreting some earlier work of A Baker and G Laures. These results are then used to deduce that, for $\ell$ a prime which generates $\mathbb{Z}_{p}^{\times}$, the spectrum $Q(\ell)$ detects the $\alpha$ and $\beta$ families in the stable stems.
\end{abstract}

55Q45; 55Q51, 55N34, 11F33

\section{Introduction}

The Adams-Novikov spectral sequence

$$
\operatorname{Ext}_{B P_{*} B P}^{s, t}\left(B P_{*}, B P_{*}\right) \Rightarrow\left(\pi_{t-s}^{S}\right)_{(p)}
$$

is one of the main tools for organizing periodic phenomena in the $p$-local stable homotopy groups of spheres. Assuming that $p$ is an odd prime, the 1-line is generated by elements

$$
\alpha_{i / j} \in \operatorname{Ext}_{B P_{*} B P}^{1,2(p-1) i-1}\left(B P_{*}, B P_{*}\right)
$$

of order $p^{j}$, for $i \geq 1$ and $j$ satisfying

$$
j=v_{p}(i)+1 .
$$

The elements $\alpha_{i / j}$ are all permanent cycles, and detect the generators of the image of the $J$-homomorphism. The image of $J$ admits a global description in terms of denominators of Bernoulli numbers: there is a correspondence

$$
\alpha_{i / j} \leftrightarrow B_{t}
$$

between the generator $\alpha_{i / j}$ and the $t$ th Bernoulli number for $t=(p-1) i$. The order $p^{j}$ of the element $\alpha_{i / j}$ is equal to the $p$-factor of the denominator of the quotient

$$
\frac{B_{t}}{t}
$$


Thus the 1-line of the Adams-Novikov spectral sequence is governed by the $p$-adic valuations of the denominators of the Bernoulli numbers. The purpose of this paper is to provide a similar description for the 2-line of the Adams-Novikov spectral sequence, in terms of certain congruences of modular forms.

Let

$$
M_{k}\left(\Gamma_{0}(N)\right)
$$

denote the space of weight $k$ modular forms for $\Gamma_{0}(N)$ defined over $\mathbb{Z}$. For a ring $R$, let

$$
M_{k}\left(\Gamma_{0}(N)\right)_{R}=M_{k}\left(\Gamma_{0}(N)\right) \otimes R
$$

be the corresponding space of modular forms defined over $R$. If $N=1$, we shall simplify the notation:

$$
\left(M_{k}\right)_{R}:=M_{k}\left(\Gamma_{0}(1)\right)_{R} .
$$

We shall sometimes work with modular forms which are simply meromorphic at $\infty$, which we shall denote

$$
M_{k}\left(\Gamma_{0}(N)\right)_{R}^{0}=M_{k}\left(\Gamma_{0}(N)\right)_{R}\left[\Delta^{-1}\right]
$$

where $\Delta \in M_{12}$ is the discriminant.

Remark 1.1 Implicit in our definition of the notation $M_{k}\left(\Gamma_{0}(N)\right)_{R}$ given by (1-1) is a non-trivial base change theorem. One typically requires $N$ to be invertible in $R$, and then one regards the modular forms for a ring $R$ as the sections of a certain line bundle of the base-change of the moduli stack of elliptic curves to $R$. In most instances considered in this paper, these two notions agree, see Katz [12, Sections 1.7,1.8].

The $q$-expansion gives rise to an embedding

$$
\begin{aligned}
M_{k}\left(\Gamma_{0}(N)\right) & \hookrightarrow \mathbb{Z}[[q]] \\
f & \mapsto f(q)
\end{aligned}
$$

and consequently embeddings

$$
\begin{aligned}
& M_{k}\left(\Gamma_{0}(N)\right)_{R} \hookrightarrow R[[q]], \\
& M_{k}\left(\Gamma_{0}(N)\right)_{R}^{0} \hookrightarrow R((q)) .
\end{aligned}
$$

Therefore, a modular form over $R$ is determined by its weight and its $q$-expansion. For any $f \in\left(M_{k}\right)_{R}$, and any prime $\ell$, the power series

$$
\left(V_{\ell} f\right)(q):=f\left(q^{\ell}\right)
$$


is the $q$-expansion of a modular form

$$
V_{\ell} f \in M_{k}\left(\Gamma_{0}(\ell)\right)_{R}
$$

Suppose that $p$ is a prime greater than 3. Miller, Ravenel and Wilson showed that the 2 -line of the Adams-Novikov spectral sequence is generated by elements

$$
\beta_{i / j, k} \in \operatorname{Ext}_{B P_{*} B P}^{2, *}\left(B P_{*}, B P_{*}\right)
$$

for $i, j$, and $k$ satisfying certain elaborate conditions (see Theorem 11.2). Our main theorem is stated below.

Theorem 1.2 For each additive generator

$$
\beta_{i / j, k} \in \operatorname{Ext}_{B P_{*} B P}^{2, *}\left(B P_{*}, B P_{*}\right)
$$

there is an associated modular form

$$
f_{i / j, k} \in M_{t}
$$

(where $\left.t=i\left(p^{2}-1\right)\right)$ satisfying:

(1) The $q$-expansion $f_{i / j, k}(q)$ is not congruent to $0 \bmod p$.

(2) We have ord $q f_{i / j, k}(q)>\frac{t-j(p-1)}{12}$ or $\operatorname{ord}_{q} f_{i / j, k}(q)=\frac{t-j(p-1)-2}{12}$.

(3) There does not exist a form

$$
g \in M_{t^{\prime}}, \text { for } t^{\prime}<t,
$$

satisfying

$$
f_{i / j, k}(q) \equiv g(q) \bmod p^{k} .
$$

(4) For every prime $\ell \neq p$, there exists a form

$$
g_{\ell} \in M_{t-j(p-1)}\left(\Gamma_{0}(\ell)\right)
$$

satisfying

$$
f_{i / j, k}\left(q^{\ell}\right)-f_{i / j, k}(q) \equiv g_{\ell}(q) \bmod p^{k}
$$

The congruence conditions met by the forms $f_{i / j, k}$ are sharp; we have the following converse theorem.

Theorem 1.3 Suppose there exists a modular form

$$
f \in M_{t}
$$


satisfying Theorem $1.2(1)-(4)$, where $t \equiv 0 \bmod (p-1) p^{k-1}$. Then $t=i\left(p^{2}-1\right)$ for some $i$, and if $i$ is not a power of $p$, there is a corresponding generator

$$
\beta_{i / j, k} \in \operatorname{Ext}_{B P_{*} B P}^{2, *}\left(B P_{*}, B P_{*}\right) .
$$

Finally, the congruence condition given in Theorem 1.2 exhibits a certain rigidity, as explained in the following theorem.

Theorem 1.4 Suppose that $\ell_{0}$ is a prime which generates $\mathbb{Z}_{p}^{\times}$. Then, if $f$ is a modular form of weight $t \equiv 0 \bmod (p-1) p^{k-1}$ satisfying Theorem 1.2 (1)-(3), and Theorem 1.2 (4) for $\ell=\ell_{0}$, then $f$ satisfies Theorem 1.2 (4) for all primes $\ell \neq p$.

Remark 1.5 In [15], G Laures introduced the $f$-invariant, a higher analog of the Adams $e$-invariant, which gives an injection of the groups $\operatorname{Ext}_{B P_{*} B P}^{2, *}\left(B P_{*}, B P_{*}\right)$ into Katz's ring of divided congruences, tensored with $\mathbb{Q} / \mathbb{Z}$. Laures' $f$-invariant therefore associates to an element $\beta_{i / j, k}$, a non-congruence between modular forms. It is natural to ask what relation this non-congruence has to the non-congruences given by condition (3) of Theorem 1.2. Laures and the author have discovered that the $f$-invariant of $\beta_{i / j, k}$ is precisely represented by the modular forms $f_{i / j, k}$ (Behrens-Laures [5]). Thus, Theorem 1.3 gives a precise description of the image of the $f$-invariant. The image of the elements $\beta_{i}$ under the $f$-invariant has been calculated in a different way by J Hornbostel and N Naumann [11].

The proofs of Theorems 1.2-1.4 use the spectrum $Q(\ell)$ introduced by the author in [3; 2]. Analyzing the chromatic spectral sequence

$$
E_{1}^{s, t}=\pi_{t} M_{s} Q(\ell) \Rightarrow \pi_{t-s} Q(\ell),
$$

we observe that a density result (Behrens-Lawson [7]) relates part of the 2-line of the chromatic spectral sequence of $Q(\ell)$ to the 2-line of the chromatic spectral sequence for the sphere. We also analyze the 0 and $1-$ lines of the chromatic spectral sequence of $Q(\ell)$, and find the following.

(1) The 0 -line $\pi_{t} M_{0} Q(\ell)$ is concentrated in $t=0,-1,-2$ (Corollary 8.4).

(2) The 1 -line $\pi_{t} M_{1} Q(\ell)$ is generated in degrees congruent to $0 \bmod 4$ by the images of the elements $\alpha_{i / j} \in \pi_{*} M_{1} S$ (Corollary 9.7).

In fact, the additive generators of $\pi_{4 t} Q(\ell)$ are given by the Eisenstein series $E_{2 t} \in$ $\left(M_{2 t}\right)_{\mathbb{Q}} 9.5$, and the orders of the groups $\pi_{4 t} M_{1} Q(\ell)$ are directly linked to the $p$-adic valuation of the denominators of the Bernoulli numbers $\frac{B_{2 t}}{4 t}$ through the appearance of the Bernoulli numbers in the $q$-expansions of the Eisenstein series. The relationship 
was originally made precise by G Laures [14], and rederived by A Baker in [1], where Hecke operations are used to conclude that Eisenstein series generate the 1-line of the TMF-Adams Novikov spectral sequence. Our analysis is closely related to these.

As a consequence of our study of the chromatic spectral sequence for $Q(\ell)$ we are able to prove the following theorem.

Theorem (Theorem 12.1) The images of the elements $\alpha_{i / j}$ and the elements $\beta_{i / j, k}$ under the homomorphism

$$
\pi_{*} S_{E(2)} \rightarrow \pi_{*} Q(\ell)
$$

are non-trivial.

This theorem shows that the homotopy of the spectrum $Q(\ell)$ is closely tied to Greek letter phenomena. It also gives credibility to the author's hope that the following questions have affirmative answers.

(1) Are the homotopy Greek letter elements $\beta_{i / j, k}^{h}$ (see the author's [4]) detected by the spectra $Q(\ell)$ at the primes 2 and 3 ?

(2) Do the spectra $Q_{U}\left(K^{p, \ell}\right)$ (constructed using Shimura varieties of type $U(1, n-$ 1) in Behrens-Lawson [6]) detect the $v_{n}$-periodic Greek letter elements?

Organization of the paper In Section 2 we summarize the chromatic spectral sequence. We also recall Morava's change of rings theorem, which relates the terms of the chromatic spectral sequence to the cohomology of the Morava stabilizer groups $\mathbb{S}_{n}$.

In Section 3 we explain how to associate a $p$-complete TMF-spectrum to every compact open subgroup of the adele group $G L_{2}\left(\mathbb{A}^{p, \infty}\right)$, following standard conventions used in the theory of modular forms. Certain $E_{\infty}$-operations between these spectra are given by elements of $G L_{2}\left(\mathbb{A}^{p, \infty}\right)$.

In Section 4 we explain how to use the $G L_{2}$ action of Section 3 to define spectra $Q(S)$ for a set of primes $S$. These spectra agree with the spectra $Q(\ell)$ defined by the author in $[3 ; 2]$ when $S=\{\ell\}$. The approach of this paper, however, mimics that of [6]. We explain how the results of [3;7] arise in this framework. In particular, we identify the $K(2)$-localization of $Q(S)$ as the homotopy fixed points of a dense subgroup $\Gamma_{S}$ of the Morava stabilizer group $\mathbb{S}_{2}$.

In Section 5 we explain how the building resolution of $Q(\ell)$ given in [3] can be recovered using the methods of [6]. We use this resolution to define a finite cochain complex $C^{\bullet}(\ell)$ of modular forms whose cohomology gives $\pi_{*} Q(\ell)$. 
In Section 6 we express the first differential in $C^{\bullet}(\ell)$ in terms of the Verschiebung of modular forms.

In Section 7 we describe the chromatic spectral sequence of $Q(\ell)$. In particular, we show that its $E_{1}$-term consists of three lines: $M_{0} Q(\ell), M_{1} Q(\ell)$, and $M_{2} Q(\ell)$. We explain how to modify the chain complex $C^{\bullet}(\ell)$ to compute these terms. We also relate $M_{2} Q(\ell)$ to the cohomology of the group $\Gamma_{\ell}$.

In Section 8 we show that $\pi_{t} M_{0} Q(\ell)$ is concentrated in $t=0,-1,-2$. We also deduce that the rest of $\pi_{t} Q(\ell)$ is $p$-torsion, and give bounds for the torsion.

In Section 9 we compute $\pi_{4 t} M_{1} Q(\ell)$, and show that its generators can be expressed as Eisenstein series. The orders of these groups are given by the $p$-adic valuation of the Bernoulli numbers $B_{2 t} / 4 t$.

In Section 10 we recall theorems of Serre and Swinnerton-Dyer, which relate congruences amongst $q$-expansions of modular forms to multiplication by the Hasse invariant $E_{p-1}$.

In Section 11 we analyze $\pi_{*} M_{2} Q(\ell)$, and prove Theorems 1.2-1.4.

In Section 12 we deduce Theorem 12.1 from our extensive knowledge of the chromatic spectral sequence for $Q(\ell)$.

Acknowledgements This paper was prepared while the author visited Harvard University, and the author is grateful for their hospitality. The author is also grateful to Niko Naumann for pointing out an error in an earlier draft concerning the integrality of Eisenstein series.

The author was supported by the NSF grant DMS-0605100, the Sloan Foundation, and DARPA.

\section{The chromatic spectral sequence}

Let $X$ be a spectrum. The chromatic tower for $X$ is the tower of Bousfield localizations with respect to the Johnson-Wilson spectra $E(n)$.

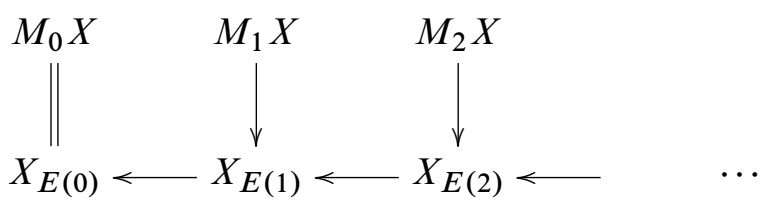


Here the fibers $M_{n} X$ are the $n$th monochromatic layers. They admit a presentation as

$$
M_{n} X \simeq \underset{I}{\operatorname{hocolim}} M(I)^{0} \wedge X_{E(n)}
$$

where $M(I)^{0}=M\left(i_{0}, \ldots, i_{n-1}\right)^{0}$ is the generalized Moore spectrum with top cell in dimension 0 and $B P$-homology,

$$
B P_{*} M\left(i_{0}, \ldots, i_{n-1}\right)=\Sigma^{-\|I\|-n} B P_{*} /\left(p^{i_{0}}, v_{1}^{i_{1}}, \ldots, v_{n-1}^{i_{n-1}}\right)
$$

with

$$
\|I\|:=\sum_{j} 2 i_{j}\left(p^{j}-1\right)
$$

and $I$ ranges over a suitable cofinal collection of multi-indices. (This colimit presentation of the monochromatic layers was conjectured in Ravenel [18, Section 5.9]. The conjecture was resolved by the Hopkins-Smith periodicity theorem [10], which implies the existence of such a system of generalized Moore spectra.)

Applying homotopy to the chromatic tower yields the chromatic spectral sequence

$$
E_{1}^{n, k}=\pi_{k} M_{n} X \Rightarrow \pi_{k-n} X_{(p)}
$$

which is conditionally convergent if $X$ is harmonic.

Morava's change of rings theorem [17] states that the Adams-Novikov spectral sequence for $M_{n} X$ takes the form

$$
H_{c}^{s}\left(\mathbb{S}_{n},\left(M_{n} E_{n}\right)_{t}(X)\right)^{\mathrm{Gal}\left(\mathbb{F}_{p}\right)} \Rightarrow \pi_{t-s}\left(M_{n} X\right) .
$$

For $p \gg n$ this spectral sequence is known to collapse. A simple instance of this (for $X=S$ ) is given by the following lemma.

Lemma 2.1 For $X=S$ and $2 p-2 \geq \max \left\{n^{2}, 2 n+2\right\}$, the spectral sequence (2-2) collapses: the groups

$$
H_{c}^{s}\left(\mathbb{S}_{n}, \pi_{t} M_{n} E_{n}\right)^{\mathrm{Gal}\left(\mathbb{F}_{p}\right)}
$$

are zero unless $t \equiv 0 \bmod 2(p-1)$.

Proof The action of an element $a$ of the finite subgroup $\mathbb{F}_{p}^{\times} \subset \mathbb{S}_{n}$ on

$$
\pi_{2 t} M_{n} E_{n} \cong\left(\pi_{2 t} E_{n}\right) /\left(p^{\infty}, v_{1}^{\infty}, \ldots, v_{n-1}^{\infty}\right) .
$$

is given by multiplication by $\widetilde{a}^{t}$, where $\widetilde{a}$ is the image of $a$ under the Teichmüller embedding $\mathbb{F}_{p}^{\times} \subset \mathbb{Z}_{p}^{\times}$. Since $\mathbb{F}_{p}^{\times}$is cyclic of order $p-1$, it follows that $\mathbb{F}_{p-1}$ acts 
trivially if and only if $t \equiv 0 \bmod p-1$. Because the subgroup $\mathbb{F}_{p}^{\times} \subset \mathbb{S}_{n}$ is central and Galois invariant, it follows that there is an induced action of $\mathbb{F}_{p}^{\times}$on

$$
H_{c}^{s}\left(\mathbb{S}_{n}, \pi_{2 t} M_{n} E_{n}\right)^{\mathrm{Gal}\left(\mathbb{F}_{p}\right)} .
$$

However, the induced action on cohomology must be trivial, because the action is obtained by restriction from the action of $\mathbb{S}_{n}$. Thus, the cohomology groups must be trivial, except when $t \equiv 0 \bmod p-1$. The result follows from the fact that if $n<p-1$, the group $\mathbb{S}_{n}$ has cohomological dimension $n^{2}$ [17].

The sparsity of the spectral sequence (2-2), together with the fact that $E_{2}^{s, t}=0$ for $s \leq n^{2}$ implies the following corollary.

Corollary 2.2 For $2(p-1) \geq \max \left\{n^{2}, 2(n+1)\right\}$, we have

$$
\pi_{t} M_{n} S \cong H_{c}^{s}\left(\mathbb{S}_{n}, \pi_{t+s} M_{n} E_{n}\right)^{\mathrm{Gal}\left(\mathbb{F}_{p}\right)}
$$

where $t=2 k(p-1)-s$ and $0 \leq s<2(p-1)$.

\section{Adelic formulation of TMF}

Let $\mathbb{A}$ denote the rational adeles. For a set of finite places $S$ of $\mathbb{Q}$, define

$$
\begin{aligned}
\widehat{\mathbb{Z}}^{S} & :=\prod_{\ell \notin S} \mathbb{Z}_{\ell}, \\
\mathbb{A}^{S, \infty} & :=\widehat{\mathbb{Z}}^{S} \otimes \mathbb{Q} .
\end{aligned}
$$

Fix a rank 2 module:

$$
V^{p}:=\mathbb{A}^{p, \infty} \oplus \mathbb{A}^{p, \infty},
$$

and let $L^{p}=\widehat{\mathbb{Z}}^{p} \oplus \widehat{\mathbb{Z}}^{p}$ be the canonical lattice contained in $V^{p}$.

For an elliptic curve $C$ over an algebraically closed field $k$ of characteristic unequal to $\ell$, let

$$
T_{\ell}(C):={\underset{\lim }{k}} C(k)\left[\ell^{k}\right]
$$

denote the $\ell$-adic Tate module of $C$. The Tate module $T_{\ell}(C)$ is a free $\mathbb{Z}_{\ell}$-module of rank 2 (Silverman [19]). If the characteristic of $k$ is zero or $p$, the $\ell$-adic Tate modules assemble to give the $\mathbb{A}^{p, \infty}$-module

$$
V^{p}(C):=T^{p}(C) \otimes \mathbb{Q},
$$


where

$$
T^{p}(C):=\prod_{\ell \neq p} T_{\ell}(C)
$$

There is a canonical short exact sequence

$$
T^{p}(C) \rightarrow V^{p}(C) \stackrel{u}{\rightarrow} C\left[\operatorname{tor}^{p}\right]
$$

where $C\left[\operatorname{tor}^{p}\right]$ is the subgroup of the group of $k$-points of $C$ consisting of those points which are torsion of order prime to $p$.

A rational uniformization is an isomorphism

$$
\eta: V^{p} \stackrel{\cong}{\rightarrow} V^{p}(C) \text {. }
$$

The group $G L_{2}\left(\mathbb{A}^{p, \infty}\right)$ acts on the set of rational uniformizations by precomposition. As explained in Behrens-Lawson [6, Section 3.2], a rational uniformization $\eta$ of $V^{p}(C)$ gives rise to a prime-to- $p$ quasi-isogeny

$$
\phi_{\eta}: C \rightarrow C_{\eta}
$$

(up to isomorphism of $C_{\eta}$ ). If the uniformization has the property that

$$
T^{p}(C) \subseteq \eta\left(L^{p}\right),
$$

the quasi-isogeny is an isogeny. In this case, the (isomorphism class of the) isogeny $\phi_{\eta}$ is characterized by its kernel $H_{\eta}$, which is given by:

$$
\operatorname{ker}(\phi)=H_{\eta}:=\operatorname{image}\left(L^{p} \hookrightarrow V^{p} \stackrel{\eta}{\rightarrow} V^{p}(C) \stackrel{u}{\rightarrow} C\left[\operatorname{tor}^{p}\right]\right)
$$

(The case of more general $\eta$, not satisfying (3-2), is easily generalized from this, producing quasi-isogenies $\phi_{\eta}$ which need not be isogenies.)

For a subgroup

$$
K^{p} \subset G L_{2}\left(\mathbb{A}^{p, \infty}\right),
$$

we let $[\eta]_{K^{p}}$ denote the $K^{p}$-orbit of rational uniformizations generated by $\eta$. The we shall refer to the orbit $[\eta]_{K^{p}}$ as an $K^{p}$-level structure. If we define

$$
K_{0}^{p}:=G L_{2}\left(\widehat{\mathbb{Z}}^{p}\right) \subset G L_{2}\left(\mathbb{A}^{p, \infty}\right),
$$

then, given a rational uniformization

$$
\eta: V^{p} \stackrel{\cong}{\rightarrow} V^{p}(C)
$$

the isomorphism class of the quasi-isogeny $\phi_{\eta}$ depends only on the $K_{0}^{p}$ level structure $[\eta]_{K_{0}^{p}}$ 
If $C$ is an elliptic scheme over a connected base $S$, we can pick a geometric point $s$ of $S$ and talk about level structures of the fiber $C_{s}$, provided they are $\pi_{1}(S, s)-$ invariant. Given a $\pi_{1}(S, s)$-invariant $K_{0}^{p}$-level structure $[\eta]_{K_{0}^{p}}$ represented by a rational uniformization

$$
\eta: V^{p} \stackrel{\eta}{\rightarrow} V^{p}\left(C_{s}\right)
$$

(satisfying (3-2)) there is an associated subgroup

$$
H_{\eta, s}<C_{s}\left[\operatorname{tor}^{p}\right]
$$

The $\pi_{1}(S, s)$-invariance of $[\eta]_{K_{0}^{p}}$ implies that $H_{\eta, s}$ extends to a local system over $S$, giving a subgroup

$$
H_{\eta}<C,
$$

and a corresponding isogeny

$$
\phi_{\eta}: C \rightarrow C / H_{\eta}=: C_{\eta} .
$$

Extending this to $\eta$ not satisfying (3-2) associates to a $\pi_{1}(S, s)$-invariant $K_{0}^{p}$-level structure $[\eta]_{K_{0}^{p}}$ of $C_{S}$ an quasi-isogeny $\phi_{\eta}: C \rightarrow C_{\eta}$ of elliptic schemes over $S$.

Associated to a compact open subgroup

$$
K^{p} \subset G L_{2}\left(\mathbb{A}^{p, \infty}\right)
$$

is a Deligne-Mumford stack $\mathcal{M}\left(K^{p}\right)$ over $\mathbb{Z}_{(p)}$ of elliptic curves with $K^{p}$-level structure (see, for instance, Hida [9, Section 7.1.2], specialized to the group $G L_{2}$ ). For a connected scheme $S$ over $\mathbb{Z}_{(p)}$ with a specified geometric point $s$, the $S$-points of $\mathcal{M}\left(K^{p}\right)$ are the groupoids whose objects are tuples

$$
\left(C,[\eta]_{K^{p}}\right)
$$

where:

$$
\begin{aligned}
C & =\text { elliptic scheme over } S, \\
{[\eta]_{K^{p}} } & =\pi_{1}(S, s) \text { invariant } K^{p} \text {-level structure on } C_{S} .
\end{aligned}
$$

The morphisms of the groupoid of $S$-points of $\mathcal{M}\left(K^{p}\right)$

$$
\alpha:\left(C,[\eta]_{K^{p}}\right) \rightarrow\left(C^{\prime},\left[\eta^{\prime}\right]_{K^{p}}\right)
$$

are the prime-to- $p$ quasi-isogenies

$$
\alpha: C \rightarrow C^{\prime}
$$

for which

$$
\left[\alpha_{*} \circ \eta\right]_{K^{p}}=\left[\eta^{\prime}\right]_{K^{p}} .
$$




\section{Remark 3.1}

(1) If the compact open subgroup is given by

$$
K_{0}^{p}:=G L_{2}\left(\widehat{\mathbb{Z}}^{p}\right) \subset G L_{2}\left(\mathbb{A}^{p, \infty}\right)
$$

then there is an isomorphism

$$
\begin{aligned}
\mathcal{M}\left(K_{0}^{p}\right) & \stackrel{\cong}{\rightarrow} \mathcal{M}_{\ell} \otimes \mathbb{Z}_{(p)} \\
\left(C,[\eta]_{K_{0}^{p}}\right) & \mapsto C_{\eta}
\end{aligned}
$$

where $\mathcal{M}_{\ell} / \mathbb{Z}$ is the usual (uncompactified) moduli stack of elliptic curves (see, for instance, Kudla [13]).

(2) If the compact open subgroup is given by

$$
K_{0}^{p}(\ell):=G L_{2}\left(\widehat{\mathbb{Z}}^{p, \ell}\right) K_{0}(\ell) \subset G L_{2}\left(\mathbb{A}^{p, \infty}\right),
$$

where $K_{0}(\ell) \subset G L_{2}\left(\mathbb{Z}_{\ell}\right)$ is the subgroup of matrices given by

$$
K_{0}(\ell)=\left\{A \in G L_{2}\left(\mathbb{Z}_{\ell}\right): A \equiv\left[\begin{array}{ll}
* & * \\
0 & *
\end{array}\right] \quad \bmod \ell\right\},
$$

then there is an isomorphism [13]

$$
\begin{aligned}
\mathcal{M}\left(K_{0}^{p}(\ell)\right) & \stackrel{\cong}{\rightarrow} \mathcal{M}\left(\Gamma_{0}(\ell)\right) \otimes_{\mathbb{Z}[1 / \ell]} \mathbb{Z}_{(p)}, \\
\left(C,[\eta]_{K_{0}^{p}(\ell)}\right) & \mapsto\left(C_{\eta}, N_{\eta}\right)
\end{aligned}
$$

where $\mathcal{M}\left(\Gamma_{0}(\ell)\right)$ is the moduli stack of elliptic curves with a $\Gamma_{0}(\ell)$-structure, and $N_{\eta}$ is the $\Gamma_{0}(\ell)$-structure (subgroup of order $\ell$ ) of $C$ associated to the image of the composite

$$
\ell^{-1} \mathbb{Z}_{\ell} \oplus \mathbb{Z}_{\ell} \rightarrow V^{p} \stackrel{\eta}{\rightarrow} V^{p}(C) \stackrel{\left(\phi_{\eta}\right)_{*}}{\longrightarrow} V^{p}\left(C_{\eta}\right) .
$$

(3) If $K_{1}^{p}<K_{2}^{p}$ is a pair of compact open subgroups, then there is an induced étale cover of moduli stacks:

$$
\begin{aligned}
\mathcal{M}\left(K_{1}^{p}\right) & \rightarrow \mathcal{M}\left(K_{2}^{p}\right), \\
\left(C,[\eta]_{K_{1}^{p}}\right) & \mapsto\left(C,[\eta]_{K_{2}^{p}}\right) .
\end{aligned}
$$

If $K_{1}^{p}$ is normal in $K_{2}^{p}$, the cover is a torsor for $K_{2}^{p} / K_{1}^{p}$.

(4) An element $g \in G L_{2}\left(\mathbb{A}^{p, \infty}\right)$ gives rise to an isomorphism of stacks

$$
\begin{aligned}
g^{*}: \mathcal{M}\left(g K^{p} g^{-1}\right) & \rightarrow \mathcal{M}\left(K^{p}\right), \\
\left(C,[\eta]_{g K^{p}} g^{-1}\right) & \mapsto\left(C,[\eta \circ g]_{K^{p}}\right) .
\end{aligned}
$$


Clearly we have

$$
g^{*}=(g k)^{*} \text { for } k \in K^{p} \text {. }
$$

The moduli of $p$-divisible groups corresponding to the moduli space $\mathcal{M}\left(K^{p}\right)$ satisfies Lurie's generalization of the Hopkins-Miller theorem [6, Section 8.1], and hence the $p$-completion $\mathcal{M}\left(K^{p}\right)_{p}^{\wedge}$ carries a presheaf of $p$-complete $E_{\infty}$-ring spectra $\mathcal{E}_{K^{p}}$ on the site $\left(\mathcal{M}\left(K^{p}\right)_{p}^{\wedge}\right)_{e t}$ such that the following hold.

(1) The presheaf $\mathcal{E}_{K^{p}}$ satisfies homotopy hyperdescent (ie it is Jardine fibrant).

(2) For an affine étale open

$$
\operatorname{Spf}(R) \stackrel{\left(C,[\eta]_{K} p\right)}{\longrightarrow} \mathcal{M}\left(K^{p}\right)_{p}^{\wedge}
$$

the corresponding spectrum of sections

$$
E=\mathcal{E}_{K^{p}}(\operatorname{Spf}(R))
$$

is a weakly even periodic elliptic spectrum associated to the elliptic curve $C$ (ie $\pi_{0}(E)=R$, and the formal group associated to $E$ is isomorphic to the formal group of $C$ ).

Define $\operatorname{TMF}\left(K^{p}\right)$ to be the global sections

$$
\operatorname{TMF}\left(K^{p}\right):=\mathcal{E}_{K^{p}}\left(\mathcal{M}\left(K^{p}\right)_{p}^{\wedge}\right)
$$

In particular, we have

$$
\begin{aligned}
\operatorname{TMF}\left(K_{0}^{p}\right) & =\operatorname{TMF}_{p}, \\
\operatorname{TMF}\left(K_{0}^{p}(\ell)\right) & =\operatorname{TMF}_{0}(\ell)_{p} .
\end{aligned}
$$

By the functoriality of Lurie's theorem [6, Section 8.1.4], the action of $G L_{2}\left(\mathbb{A}^{p, \infty}\right)$ described in Remark 3.1 gives rise to maps of $E_{\infty}$-ring spectra

$$
g_{*}: \operatorname{TMF}\left(K^{p}\right) \rightarrow \operatorname{TMF}\left(g K^{p} g^{-1}\right) .
$$

\section{The spectra $Q(S)$}

The collection of compact open subgroups $K^{p}$ of $G L_{2}\left(\mathbb{A}^{p, \infty}\right)$ under inclusion forms a filtered category, and we may take the colimit

$$
\mathcal{V}:=\underset{K^{p}}{\operatorname{colim}} \operatorname{TMF}\left(K^{p}\right) .
$$


The action of $G L_{2}\left(\mathbb{A}^{p, \infty}\right)$ described above gives $\mathcal{V}$ the structure of a smooth $G L_{2}\left(\mathbb{A}^{p, \infty}\right)$-spectrum $\left[6\right.$, Section 10.3]. We may recover each of the $\operatorname{spectra} \operatorname{TMF}\left(K^{p}\right)$ from $\mathcal{V}$ by taking homotopy fixed points [6, Section 10.6.5]:

$$
\operatorname{TMF}\left(K^{p}\right) \simeq \mathcal{V}^{h K^{p}}
$$

For a set of primes $S$ not containing $p$, we have an open subgroup

$$
\left(K_{0}^{p, S}\right)_{+}:=G L_{2}\left(\mathbb{A}_{S}\right) K_{0}^{p, S} \subset G L_{2}\left(\mathbb{A}^{p, \infty}\right)
$$

where $\mathbb{A}_{S}=\prod_{\ell \in S}^{\prime} \mathbb{Q}_{\ell}$ is the ring of $S$-adeles and

$$
K_{0}^{p, S}=\prod_{\ell \notin\{p\} \cup S} G L\left(\mathbb{Z}_{\ell}\right) .
$$

We define a spectrum

$$
Q(S)=\mathcal{V}^{h\left(K_{0}^{p, S}\right)_{+}}
$$

The $K(2)$-localization of the spectrum $Q(S)$ is closely related to the $K(2)$-local sphere, as we now explain. Let $C_{0}$ be a fixed supersingular curve over $\overline{\mathbb{F}}_{p}$ (any two are isogenous). Assume (for convenience) that $C_{0}$ is defined over $\mathbb{F}_{p}$ (such a curve exists for every prime $p$ Waterhouse [20]). The quasi-endomorphism ring

$$
D:=\operatorname{End}^{0}\left(C_{0}\right)
$$

is a quaternion algebra over $\mathbb{Q}$ ramified at $p$ and $\infty$. The subring of actual endomorphisms

$$
\mathcal{O}_{D}:=\operatorname{End}\left(C_{0}\right) \subset D
$$

is a maximal order. For our set of primes $S$, define a group

$$
\Gamma_{S}:=\left(\mathcal{O}_{D}\left[S^{-1}\right]\right)^{\times} .
$$

The group $\Gamma_{S}$ is the group of quasi-isogenies of $C_{0}$ whose degree lies in

$$
\mathbb{Z}\left[S^{-1}\right]^{\times} \subset \mathbb{Q}^{\times} .
$$

The group $\Gamma_{S}$ embeds in the (profinite) Morava stabilizer group through its action on the height 2 formal group of $C_{0}$ :

$$
\Gamma_{S} \hookrightarrow \operatorname{Aut}\left(\widehat{C}_{0}\right) \cong \mathbb{S}_{2} .
$$

Theorem 4.1 (Behrens-Lawson [7]) If $p$ is odd, and $S$ contains a generator of $\mathbb{Z}_{p}^{\times}$, then the subgroup

$$
\Gamma_{S} \hookrightarrow \mathbb{S}_{2}
$$

is dense. 
The universal deformation $\widetilde{C}_{0}$ of the supersingular curve $C_{0}$, by Serre-Tate theory, gives Morava $E$-theory $E_{2}$ the structure of an elliptic spectrum, where

$$
\pi_{*} E_{2}=W\left(\overline{\mathbb{F}}_{p}\right)\left[\left[u_{1}\right]\right]\left[u^{ \pm 1}\right] .
$$

Since $C_{0}$ is assumed to admit a definition over $\mathbb{F}_{p}$, there is an action of the Galois group $\operatorname{Gal}\left(\mathbb{F}_{p}\right)$ on the spectrum $E_{2}$. Picking a fixed rational uniformization

$$
\eta_{0}: V^{p} \stackrel{\cong}{\rightarrow} V^{p}\left(C_{0}\right)
$$

gives, for every $K^{p}$, a canonical map of $E_{\infty}$-ring spectra

$$
\operatorname{TMF}\left(K^{p}\right) \stackrel{\left(\widetilde{C}_{0,}\left[\eta_{0}\right]_{K}\right)}{\longrightarrow} E_{2}
$$

classifying the pair $\left(\widetilde{C}_{0},\left[\eta_{0}\right]_{K^{p}}\right)$, thus a map

$$
\mathcal{V} \stackrel{\left(\widetilde{C}_{0}, \eta_{0}\right)}{\longrightarrow} E_{2}
$$

Using the Tate embedding

$$
\operatorname{End}^{0}\left(C_{0}\right) \hookrightarrow \operatorname{End}\left(V^{p}\left(C_{0}\right)\right),
$$

the rational uniformization $\eta_{0}$ induces an inclusion

$$
\begin{aligned}
\gamma: \Gamma_{S} \hookrightarrow\left(K_{0}^{p, S}\right)_{+} \subseteq G L_{2}\left(\mathbb{A}^{p, \infty}\right), \\
\alpha \mapsto \eta_{0}^{-1} \alpha \eta_{0} .
\end{aligned}
$$

\section{Lemma 4.2}

(1) For $\alpha \in \Gamma_{S}$, the following diagram commutes

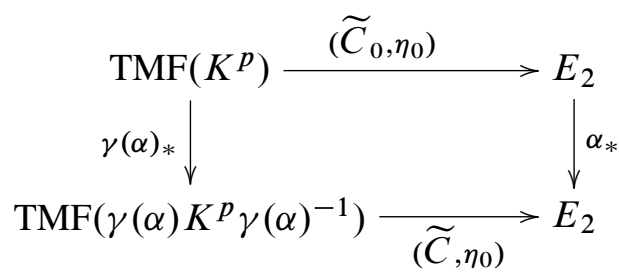

where $\gamma(\alpha)_{*}$ is the morphism induced by the action of $G L_{2}\left(\mathbb{A}^{p, \infty}\right)$ on TMF, and $\alpha_{*}$ is the morphism induced by the action of the Morava stabilizer group on $E_{2}$ through the inclusion $\Gamma_{S} \subset \mathbb{S}_{2}$.

(2) The map

$$
\operatorname{TMF}\left(K^{p}\right) \stackrel{\left(\widetilde{C}_{0}, \eta_{0}\right)}{\longrightarrow} E_{2}
$$

is invariant under the action of $\mathrm{Gal}\left(\mathbb{F}_{p}\right)$ on $E_{2}$. 
Proof Let $\operatorname{Def}\left(C_{0}\right)$ denote the formal moduli of deformations of $C_{0}$. For a complete local ring $(R, \mathfrak{m})$, the $R$-points of $\operatorname{Def}\left(C_{0}\right)$ is the category of tuples

$$
(C, \iota, \beta) \text {, }
$$

where

$$
\begin{aligned}
& C=\text { elliptic curve over } R, \text { with reduction } \bar{C} \bmod \mathfrak{m}, \\
& \iota: \overline{\mathbb{F}} p \rightarrow R / \mathfrak{m}, \\
& \beta: \iota^{*} C_{0} \cong \bar{C} .
\end{aligned}
$$

The element $\alpha \in \Gamma_{S}$ acts on the $R$-points of $\operatorname{Def}\left(C_{0}\right)$ by

$$
\alpha^{*}:(C, \iota, \beta) \mapsto(C, \iota, \beta \circ \alpha) .
$$

By Serre-Tate theory, this space is equivalent to the formal moduli $\operatorname{Def}\left(\widehat{C}_{0}\right)$ of deformations of the associated height 2 formal group $\widehat{C}_{0}$, and the action of $\Gamma_{S}$ on $\operatorname{Def}\left(C_{0}\right)$ is compatible with the action of $\mathbb{S}_{2}$ on $\operatorname{Def}\left(\widehat{C}_{0}\right)$. Part (1) of the lemma follows from the commutativity of the following diagram, which is easily checked on $R$-points.

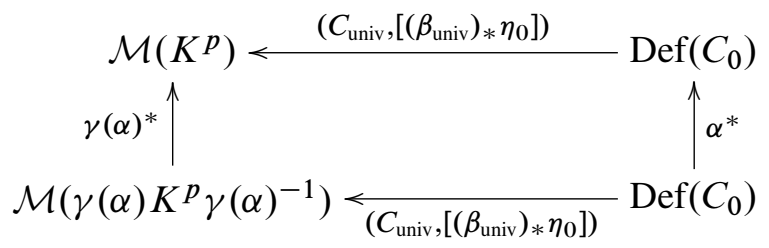

Part (2) is checked in a similar manner.

Lemma 4.2 implies that the morphism (4-2) descends to give a morphism

$$
Q(S)=\mathcal{V}^{h\left(K^{p, S}\right)_{+}} \rightarrow\left(E_{2}^{h \Gamma_{S}}\right)^{h \mathrm{Fr}_{p}}=: E\left(\Gamma_{S}\right) .
$$

Here, if $X$ is a spectrum with an action of the Frobenius $\operatorname{Fr}_{p} \in \operatorname{Gal}\left(\mathbb{F}_{p}\right)$, the spectrum $X^{h \operatorname{Fr}_{p}}$ is defined to be the homotopy fiber

$$
X^{h \mathrm{Fr}_{p}} \rightarrow X \stackrel{\mathrm{Fr}_{p}-1}{\longrightarrow} X .
$$

The following theorem is proved in [3] in the case where $S$ consists of one prime. The proof of the more general case is identical to the proof of [6, Corollary 14.5.6].

Theorem 4.3 The map (4-3) induces an equivalence

$$
Q(S)_{K(2)} \rightarrow E\left(\Gamma_{S}\right)
$$




\section{The building resolution}

If $S=\{\ell\}$ is a set containing one prime, the spectrum $Q(\ell)$ defined in Section 4 is equivalent to the spectrum constructed in [3]. In [3], the spectrum $Q(\ell)$ was defined to be the totalization of a certain semi-cosimplicial $E_{\infty}$-ring spectrum. This description is recovered as follows.

The group $G L_{2}\left(\mathbb{Q}_{\ell}\right)$ acts on its building $\mathcal{B}=\mathcal{B}\left(G L_{2}\left(\mathbb{Q}_{\ell}\right)\right)$ with compact open stabilizers. Explicitly, the building $\mathcal{B}$ is equivariantly homeomorphic to the geometric realization of a semi-simplicial $G L_{2}\left(\mathbb{Q}_{\ell}\right)$-set $\mathcal{B} \bullet$ of the form

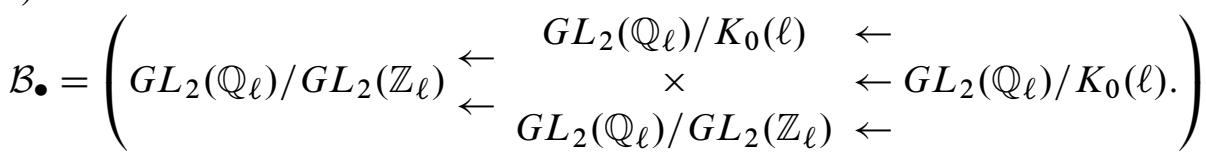

The action of $G L_{2}\left(\mathbb{Q}_{\ell}\right)$ on the building $\mathcal{B}$ extends to an action of $\left(K_{0}^{p, \ell}\right)_{+}$, simply by letting the local factors away from $\ell$ act trivially. Regarded as a semi-simplicial $\left(K_{0}^{p, \ell}\right)_{+}-$set, we have

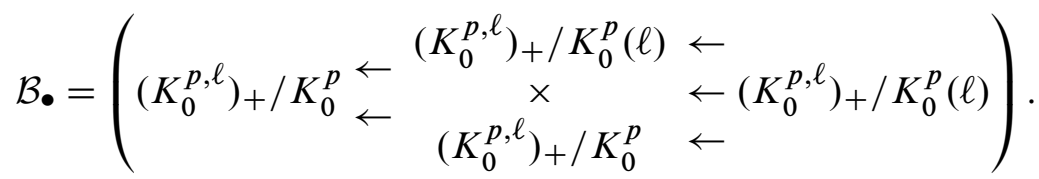

The canonical $\left(K_{0}^{p, \ell}\right)_{+}$-equivariant morphism

$$
\mathcal{V} \stackrel{c}{\rightarrow} \operatorname{Map}(\mathcal{B}, \mathcal{V})^{s m}
$$

(given by the inclusion of the constant functions) is an equivalence [6, Lemma 13.2.3]. Here, Map $(-,-)^{s m}$ is defined to be the colimit of the $U$-fixed point spectra, as $U$ ranges over the open subgroups of $G L_{2}\left(\mathbb{A}^{p, \infty}\right)$. The argument in [6] relies on the fact that the building $\mathcal{B}$ is not only contractible, but possesses a canonical contracting homotopy with excellent equivariance properties.

The semi-simplicial decomposition of $\mathcal{B}$ induces an equivariant equivalence

$$
\underline{\operatorname{Map}}(\mathcal{B}, \mathcal{V})^{s m} \simeq \operatorname{Tot} \underline{\operatorname{Map}}\left(\mathcal{B}_{\bullet}, \mathcal{V}\right)^{s m}
$$

and therefore an equivalence on fixed point spectra:

$$
\begin{aligned}
Q(\ell) & =\mathcal{V}^{h\left(K_{0}^{p, \ell}\right)_{+}} \\
& \simeq \operatorname{Tot}\left(\underline{\operatorname{Map}}\left(\mathcal{B}_{\bullet}, \mathcal{V}\right)^{s m}\right)^{h\left(K_{0}^{p, \ell}\right)_{+}} .
\end{aligned}
$$


Using Shapiro's lemma in the context of smooth $\left(K_{0}^{p, \ell}\right)_{+}-$spectra gives an equivalence

$$
\left.\underline{(\operatorname{Map}}\left(\left(K_{0}^{p, \ell}\right)_{+} / K^{p}, \mathcal{V}\right)^{s m}\right)^{h\left(K_{0}^{p, \ell}\right)_{+}} \simeq \mathcal{V}^{h K^{p}}
$$

Since we have

$$
\begin{gathered}
\mathcal{V}^{h K_{0}^{p}} \simeq \operatorname{TMF}\left(K_{0}^{p}\right)=\operatorname{TMF}_{p} \\
\mathcal{V}^{h K_{0}^{p}(\ell)} \simeq \operatorname{TMF}\left(K_{0}^{p}(\ell)\right)=\operatorname{TMF}_{0}(\ell)_{p} .
\end{gathered}
$$

Thus there is an induced semi-cosimplicial decomposition

$$
Q(\ell) \simeq \operatorname{Tot} Q(\ell)^{\bullet}
$$

where

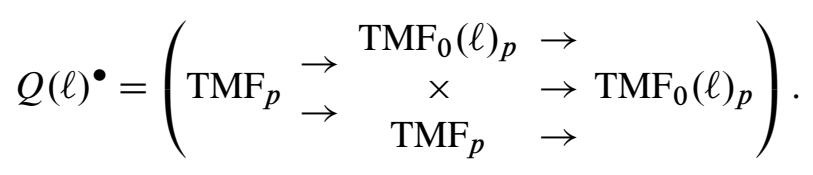

For $p \geq 5$, the homotopy groups of $\mathrm{TMF}_{p}$ and $\mathrm{TMF}_{0}(\ell)_{p}$ are concentrated in even degrees, and there are isomorphisms

$$
\begin{aligned}
\pi_{2 k} \mathrm{TMF}_{p} & \cong\left(M_{k}\right)_{\mathbb{Z}_{p}}^{0}, \\
\pi_{2 k} \mathrm{TMF}_{0}(\ell)_{p} & \cong M_{k}\left(\Gamma_{0}(\ell)\right)_{\mathbb{Z}_{p}}^{0} .
\end{aligned}
$$

Applying homotopy to the semi-cosimplicial spectrum $Q(\ell)^{\bullet}(5-5)$ gives a semicosimplicial abelian group

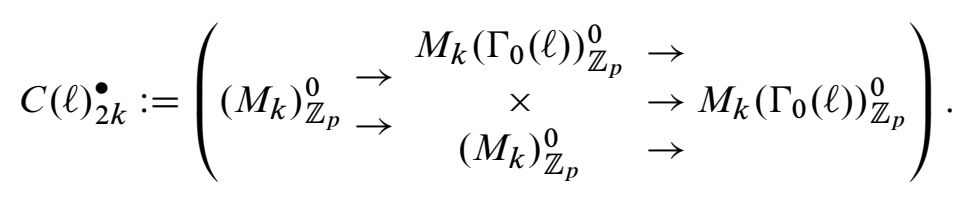

The Bousfield-Kan spectral sequence for $Q(\ell)^{\bullet}$ takes the form

$$
E_{1}^{s, t}=C(\ell)_{t}^{s} \Rightarrow \pi_{t-s} Q(\ell)
$$

Proposition 5.1 For $p \geq 5$, the spectral sequence (5-7) collapses at $E_{2}$ to give an isomorphism

$$
\pi_{n} Q(\ell) \cong H^{0}\left(C(\ell)_{n}^{\bullet}\right) \oplus H^{1}\left(C(\ell)_{n+1}^{\bullet}\right) \oplus H^{2}\left(C(\ell)_{n+2}^{\bullet}\right) .
$$


Proof The rings of modular forms $M_{*}$ and $M_{*}\left(\Gamma_{0}(\ell)\right)$ are concentrated in even weights. This easily follows in the case of $\Gamma_{0}(\ell)$ from the fact that the inversion [-1]: $C \rightarrow C$ gives an automorphism of any $\Gamma_{0}(\ell)$-structure. Thus there is no room for differentials, or hidden extensions, in the spectral sequence (5-7).

In fact, since we have argued that $H^{s}\left(C(\ell)_{t}^{\bullet}\right)$ is non-zero unless $t \equiv 0 \bmod 4$, we have the following.

Corollary 5.2 For $p \geq 5$, there are isomorphisms

$$
\pi_{t} Q(\ell) \cong H^{s}\left(C(\ell)^{\bullet}\right)_{t+s}
$$

where $t=4 k-s$ and $0 \leq s<4$.

\section{Effect of coface maps on modular forms}

Suppose that $p \geq 5$. In this section we will deduce the effect of the two initial cosimplicial coface maps of $C(\ell)_{2 k}^{\bullet}$ on the level of $q$-expansions. To aid in this, we recall from [3] that the semi-cosimplicial resolution of $Q(\ell)$ may be constructed by applying the Goerss-Hopkins-Miller presheaf to a semi-simplicial object in the site $\left(\mathcal{M}_{\ell}\right)_{e t}$ :

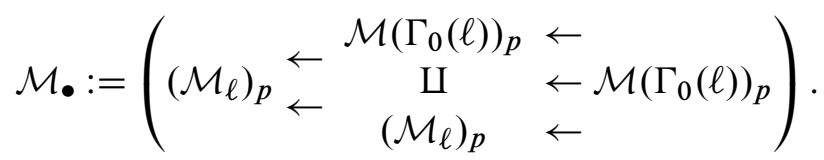

The coface maps $d_{i}: \mathcal{M}_{1} \rightarrow \mathcal{M}_{0}$ are given on $R$-points by

$$
\begin{aligned}
d_{i}:\left(\mathcal{M}_{\ell}\right)_{p} & \rightarrow\left(\mathcal{M}_{\ell}\right)_{p} \\
d_{0}: C & \mapsto C / C[\ell] \\
d_{1}: C & \mapsto C \\
d_{i}:\left(\mathcal{M}\left(\Gamma_{0}(\ell)\right)\right)_{p} & \rightarrow\left(\mathcal{M}_{\ell}\right)_{p} \\
d_{0}:(C, H) & \mapsto C / H \\
d_{1}:(C, H) & \mapsto C .
\end{aligned}
$$

Proposition 6.1 Consider the morphisms

$$
d_{0}, d_{1}:\left(M_{k}\right)_{\mathbb{Z}_{p}}^{0} \rightarrow M_{k}\left(\Gamma_{0}(\ell)\right)_{\mathbb{Z}_{p}}^{0} \times\left(M_{k}\right)_{\mathbb{Z}_{p}}^{0}
$$


induced by the initial coface maps of the cosimplicial abelian group $C(\ell)_{2 k}^{\bullet}$. On the level of $q$-expansions, the maps are given by

$$
\begin{aligned}
& d_{0}(f(q)):=\left(\ell^{k} f\left(q^{\ell}\right), \ell^{k} f(q)\right), \\
& d_{1}(f(q)):=(f(q), f(q)) .
\end{aligned}
$$

Proof It is clear from the description of the map $d_{1}$ that its effect on $q$-expansions is as given. Choosing an embedding $\mathbb{Z}_{p} \hookrightarrow \mathbb{C}$, by the $q$-expansion principle, it suffices to verify these identities hold when we base-change to $\mathbb{C}$ and consider the Tate curve:

$$
C_{q}:=\mathbb{C}^{\times} / q^{\mathbb{Z}} \text {. }
$$

The group of $\ell$ th roots of unity $\mu_{\ell} \subset \mathbb{C}^{\times}$induces a $\Gamma_{0}(\ell)$-structure on the Tate curve $C_{q}$ :

$$
\mu_{\ell} \subset \mathbb{C}^{\times} / q^{\mathbb{Z}}=C_{q}
$$

This level structure is the kernel of the isogeny

$$
\begin{aligned}
\phi_{\mu_{\ell}}: C_{q}=\mathbb{C}^{\times} / q^{\mathbb{Z}} & \rightarrow \mathbb{C}^{\times} / q^{\ell \mathbb{Z}}=C_{q^{\ell}}, \\
z & \mapsto z^{\ell} .
\end{aligned}
$$

The invariant differential $d z / z$ on $C_{q}$ transforms under this isogeny by

$$
\phi^{*}(d z / z)=\ell d z / z .
$$

It follows that $d_{0}$ on the $M_{k}\left(\Gamma_{0}(\ell)\right)_{\mathbb{Z}_{p}}^{0}$-component is given by

$$
\begin{aligned}
d_{0}:\left(M_{k}\right)_{\mathbb{Z}_{p}}^{0} & \rightarrow M_{k}\left(\Gamma_{0}(\ell)\right)_{\mathbb{Z}_{p}}^{0}, \\
f(q) & \mapsto \ell^{k} f\left(q^{\ell}\right),
\end{aligned}
$$

as desired. The $\ell$ th power map

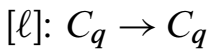

$$
\begin{aligned}
& z \mapsto z^{\ell}
\end{aligned}
$$

transforms the invariant differential by

$$
[\ell]^{*}(d z / z)=\ell d z / z .
$$

If follows that the $\left(M_{k}\right)_{\mathbb{Z}_{p}}^{0}$ component of $d_{0}$ is given by

$$
\begin{aligned}
d_{0}:\left(M_{k}\right)_{\mathbb{Z}_{p}}^{0} & \rightarrow\left(M_{k}\right)_{\mathbb{Z}_{p}}^{0}, \\
f(q) & \mapsto \ell^{k} f(q) .
\end{aligned}
$$




\section{The chromatic spectral sequence for $Q(\ell)$}

The following lemma implies that the chromatic resolution of $Q(\ell)$ is finite.

Lemma 7.1 The spectrum $Q(\ell)$ is $E(2)-$ local.

Proof The spectra $\mathrm{TMF}_{p}$ and $\mathrm{TMF}_{0}(\ell)_{p}$ are $E(2)$-local. By (5-4), the spectrum $Q(\ell)$ is $E(2)-$ local.

We deduce that the chromatic resolution for $Q(\ell)$ takes the following form.

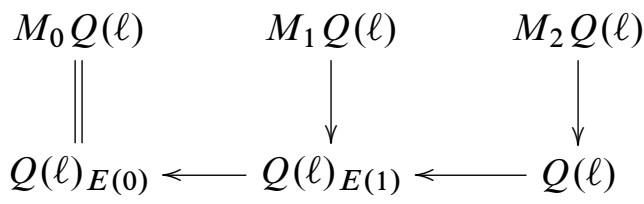

Applying homotopy, we get a three line spectral sequence

$$
E_{1}^{n, k}=\left\{\begin{array}{ll}
\pi_{k} M_{n} Q(\ell), & n \leq 2 \\
0, & n>2
\end{array}\right\} \Rightarrow \pi_{k-n} Q(\ell) .
$$

Assuming that $p \geq 5$, applying $M_{n}$ to the cosimplicial resolution (5-4), we get spectral sequences

$$
\begin{gathered}
E_{2}^{s, t}=H^{s}\left(C(\ell)^{\bullet}\left[p^{-1}\right]\right)_{t} \Rightarrow \pi_{t-s} M_{0} Q(\ell) \\
E_{2}^{s, t}=H^{s}\left(C(\ell)^{\bullet} / p^{\infty}\left[v_{1}^{-1}\right]\right)_{t} \Rightarrow \pi_{t-s} M_{1} Q(\ell) \\
E_{2}^{s, t}=H^{s}\left(C(\ell)^{\bullet} /\left(p^{\infty}, v_{1}^{\infty}\right)\right)_{t} \Rightarrow \pi_{t-s} M_{2} Q(\ell) .
\end{gathered}
$$

Here, the $E_{2}$-terms are the cohomology of the cosimplicial abelian group obtained from applying the functor $\pi_{*}\left(M_{n}-\right)$ to (5-4). The values of the resulting cosimplicial abelian group are given by the following lemma.

Lemma 7.2 Let $p \geq 5$ and $(N, p)=1$. Then

$$
\begin{aligned}
\pi_{2 *} M_{0} \mathrm{TMF}_{0}(N)_{p} & =M_{*}\left(\Gamma_{0}(N)\right)_{\mathbb{Q}_{p}}^{0}, \\
\pi_{2 *} M_{1} \mathrm{TMF}_{0}(N)_{p} & =M_{*}\left(\Gamma_{0}(N)\right)_{\mathbb{Z}_{p}}^{0} / p^{\infty}\left[E_{p-1}^{-1}\right], \\
\pi_{2 *} M_{2} \operatorname{TMF}_{0}(N)_{p} & =M_{*}\left(\Gamma_{0}(N)\right)_{\mathbb{Z}_{p}}^{0} /\left(p^{\infty}, E_{p-1}^{\infty}\right) .
\end{aligned}
$$

Proof This is a direct application of (2-1). Here, $E_{p-1}$ is the $(p-1)$ st Eisenstein series, which reduces to the Hasse invariant $v_{1} \bmod p[12$, Section 2.1]. 
In particular, since $C(\ell)_{t}^{\bullet}$ is non-zero only when $t \equiv 0 \bmod 4$, the same argument proving Corollary 5.2 gives the following.

Corollary 7.3 For $p \geq 5$, there are isomorphisms

$$
\begin{aligned}
& \pi_{t} M_{0} Q(\ell) \cong H^{s}\left(C(\ell)^{\bullet}\left[p^{-1}\right]\right)_{t+s} \\
& \pi_{t} M_{1} Q(\ell) \cong H^{s}\left(C(\ell)^{\bullet} /\left(p^{\infty}\right)\left[v_{1}^{-1}\right]\right)_{t+s} \\
& \pi_{t} M_{2} Q(\ell) \cong H^{s}\left(C(\ell)^{\bullet} /\left(p^{\infty}, v_{1}^{\infty}\right)\right)_{t+s}
\end{aligned}
$$

where $t=4 k-s$ and $0 \leq s<4$.

We end this section by relating $M_{2} Q(\ell)$ to the subgroup $\Gamma_{\ell} \subset \mathbb{S}_{n}$. By Theorem 4.3 there are equivalences

$$
M_{2} Q(\ell) \simeq M_{2}\left(Q(\ell)_{K(2)}\right) \simeq M_{2}\left(\left(E_{2}^{h \Gamma_{\ell}}\right)^{h \mathrm{Fr}_{p}}\right) .
$$

We recall the following result from [3].

Proposition 7.4 The group $\Gamma_{\ell}$ acts on the building $\mathcal{B}$ for $G L_{2}\left(\mathbb{Q}_{\ell}\right)$ with finite stabilizers, given by groups of automorphisms of supersingular curves.

We deduce the following.

Lemma 7.5 There is an equivalence

$$
M_{2}\left(\left(E_{2}^{h \Gamma_{\ell}}\right)^{h \mathrm{Fr}_{p}}\right) \simeq\left(\left(M_{2} E_{2}\right)^{h \Gamma_{\ell}}\right)^{h \mathrm{Fr}_{p}} .
$$

Proof Since the spectra $M(I)^{0}$ are finite, we have

$$
M(I)^{0} \wedge\left(\left(E_{2}^{h \Gamma_{\ell}}\right)^{h \mathrm{Fr}_{p}}\right) \simeq\left(\left(M(I)^{0} \wedge E_{2}\right)^{h \Gamma_{\ell}}\right)^{h \mathrm{Fr}_{p}} .
$$

The result would follow from (2-1) if we could commute the homotopy colimit over $I$ with the homotopy fixed points with respect to $\Gamma_{\ell}$. However, by Proposition 7.4, the group $\Gamma_{\ell}$ acts on the building $\mathcal{B}$ for $G L_{2}\left(\mathbb{Q}_{\ell}\right)$ with finite stabilizers. Since $\mathcal{B}$ is contractible and finite dimensional, we conclude that the group $\Gamma_{\ell}$ has finite virtual cohomological dimension.

We conclude that there is an equivalence

$$
M_{2} Q(\ell) \simeq\left(\left(M_{2} E_{2}\right)^{h \Gamma_{\ell}}\right)^{h \mathrm{Fr}_{p}}
$$

and a homotopy fixed point spectral sequence

$$
E_{2}^{s, t}=H^{s}\left(\Gamma_{\ell}, \pi_{t} M_{2} E_{2}\right)^{\mathrm{Gal}\left(\mathbb{F}_{p}\right)} \Rightarrow \pi_{t-s} M_{2} Q(\ell) .
$$


Lemma 7.6 For $p \geq 5$, we have:

(1) $H^{s}\left(\Gamma_{\ell}, \pi_{t} M_{2} E_{2}\right)^{\mathrm{Gal}\left(\mathbb{F}_{p}\right)}=0$ for $s>2$.

(2) $H^{s}\left(\Gamma_{\ell}, \pi_{t} M_{2} E_{2}\right)^{\mathrm{Gal}\left(\mathbb{F}_{p}\right)}=0$ for $t \not \equiv 0 \bmod 4$.

(3) There are isomorphisms

$$
\pi_{t} M_{2} Q(\ell) \cong H^{s}\left(\Gamma_{\ell}, \pi_{t+s} M_{2} E_{2}\right)^{\mathrm{Gal}\left(\mathbb{F}_{p}\right)}
$$

where $t=4 k-s$ and $0 \leq s<4$.

Proof (1) follows from Proposition 7.4, together with the fact that the coefficients are $p$-local and the building is contractible and 2-dimensional. (2) follows from the fact that there is a central, Galois invariant element $[-1] \in \Gamma_{\ell}$ (given by inversion) which acts on $\pi_{2 i} M_{2} E_{2}$ by $(-1)^{i}$. (3) follows from (1) and (2), using the spectral sequence (7-5).

Combining Corollary 7.3 with Lemma 7.6, we get the following.

Corollary 7.7 For $p \geq 5$, there are isomorphisms

$$
H^{s}\left(C(\ell)^{\bullet} /\left(p^{\infty}, v_{1}^{\infty}\right)\right)_{t} \cong H^{s}\left(\Gamma_{\ell}, \pi_{t} M_{2} E_{2}\right)^{\mathrm{Gal}\left(\mathbb{F}_{p}\right)} .
$$

Remark 7.8 One could give a purely algebraic proof of Corollary 7.7 which makes no reference to topology. In the context of the exposition of this paper it happens to be quicker (but arguably less natural) to use topological constructions.

\section{$8 \quad M_{0} Q(\ell)$}

Let $p \geq 5$ and $\ell$ be a topological generator of $\mathbb{Z}_{p}^{\times}$. In this section we will concern ourselves with locating the non-trivial homotopy of $M_{0} Q(\ell)$.

Proposition 8.1 The groups

$$
H^{S}\left(C^{\bullet}(\ell)\right)_{2 t}
$$

consist entirely if $p^{j}$-torsion if

$$
t \equiv 0 \bmod (p-1) p^{j-1},
$$

and are zero if $t \not \equiv 0 \bmod (p-1)$. 
Proof Consider the central element

$$
[\ell]:=\left[\begin{array}{ll}
\ell & 0 \\
0 & \ell
\end{array}\right] \in G L_{2}\left(\mathbb{Q}_{\ell}\right) .
$$

Let $\mathcal{V}$ be the smooth $G L_{2}\left(\mathbb{A}^{p, \infty}\right)$-spectrum of (4-1). We assume that $\mathcal{V}$ is fibrant as a smooth $G L_{2}\left(\mathbb{A}^{p, \infty}\right)$-spectrum, so that homotopy fixed points are equivalent to point-set level fixed points

$$
\mathcal{V}^{h U} \simeq \mathcal{V}^{U}
$$

for $U$ an open subgroup of $G L_{2}\left(\mathbb{A}^{p, \infty}\right)$ [6, Corollary 10.5.5]. Because [ $\left.\ell\right]$ is central, the action of $[\ell]$ on $\mathcal{V}$ is $G L_{2}\left(\mathbb{A}^{p, \infty}\right)$-equivariant. Because $[\ell]$ is contained in the subgroup $\left(K_{0}^{p, \ell}\right)_{+}$, it acts as the identity on

$$
Q(\ell) \simeq \mathcal{V}^{\left(K_{0}^{p, \ell}\right)_{+}} .
$$

However, the morphism $c$ of (5-3) is compatible with the action of $[\ell]$, where we let $[\ell]$ act on $\operatorname{Map}(\mathcal{B}, \mathcal{V})^{s m}$ through its action on the target $\mathcal{V}$. We deduce that the endomorphism $[\ell]$ acts on the cosimplicial object $Q(\ell)^{\bullet}$, where the action is given level-wise on each factor by the endomorphism

$$
[\ell]: \operatorname{TMF}\left(K^{p}\right) \rightarrow \operatorname{TMF}\left(K^{p}\right)
$$

(where $K^{p}$ is either $K_{0}^{p}$ or $K_{0}^{p}(\ell)$ ). The endomorphism $[\ell]$ is the induced action of $[\ell]$ on the fixed point spectrum

$$
\operatorname{TMF}\left(K^{p}\right) \simeq \mathcal{V}^{h K^{p}}
$$

The action of $[\ell]$ on the homotopy groups of $\operatorname{TMF}\left(K^{p}\right)$ is given by

$$
\begin{aligned}
{[\ell]: \pi_{2 k} \operatorname{TMF}\left(\Gamma_{0}(N)\right) } & \rightarrow \pi_{2 k} \operatorname{TMF}\left(\Gamma_{0}(N)\right), \\
f & \mapsto \ell^{k} f .
\end{aligned}
$$

This is easily deduced from the fact that the induced quasi-isogeny (3-1)

$$
C_{\eta} \rightarrow C_{\eta \circ[\ell]}
$$

is isomorphic to the $\ell$ th power map of elliptic curves. It follows that

$$
[\ell]: H^{s}\left(C(\ell)_{2 k}^{\bullet}\right) \rightarrow H^{s}\left(C(\ell)_{2 k}^{\bullet}\right)
$$

acts by multiplication by $\ell^{k}$. However, since we have shown that $[\ell]$ acts by the identity on $\pi_{*} Q(\ell)$, Proposition 5.1 implies that $[\ell]$ acts by the identity on $H^{s}\left(C(\ell)_{2 k}^{\bullet}\right)$. We deduce that multiplication by $\ell^{k}-1$ is the zero homomorphism on $H^{s}\left(C(\ell)_{2 k}^{\bullet}\right)$. Since $\ell$ was assumed to be a topological generator of $\mathbb{Z}_{p}^{\times}$, the proposition follows. 
We immediately deduce the following.

Corollary 8.2 We have

$$
H^{S}\left(C^{\bullet}(\ell)\left[p^{-1}\right]\right)_{t}=0
$$

for $t \neq 0$.

We can be more specific in the case of $s=0$.

\section{Lemma 8.3 We have}

$$
H^{0}\left(C^{\bullet}(\ell)\left[p^{-1}\right]\right)_{0}=\mathbb{Q}_{p}
$$

Proof We must analyze the kernel of the cosimplicial differential

$$
d_{0}-d_{1}:\left(M_{0}\right)_{\mathbb{Q}_{p}}^{0} \rightarrow M_{0}\left(\Gamma_{0}(\ell)\right)_{\mathbb{Q}_{p}}^{0} \oplus\left(M_{0}\right)_{\mathbb{Q}_{p}}^{0}
$$

We claim that is is given by the subspace generated by $1 \in\left(M_{0}\right)_{\mathbb{Q}_{p}}$. Indeed, suppose that $f \in\left(M_{0}\right)_{\mathbb{Q}_{p}}^{0}$ satisfies

$$
d_{0}(f)-d_{1}(f)=0 .
$$

By Proposition 6.1, it follows that

$$
f\left(q^{\ell}\right)-f(q)=0
$$

Writing $f(q)=\sum a_{n} q^{n}$, we find

$$
a_{n}=\left\{\begin{array}{lll}
a_{n / \ell}, & n \equiv 0 & \bmod \ell \\
0, & n \neq 0 & \bmod \ell .
\end{array}\right.
$$

It follows by induction that $f(q)=a_{0}$.

Applying this knowledge to the spectral sequence (7-2), we deduce the following.

Corollary 8.4 We have

$$
\pi_{t}\left(M_{0} Q(\ell)\right)=0
$$

if $t \notin\{0,-1,-2\}$, and

$$
\pi_{0}\left(M_{0} Q(\ell)\right)=\mathbb{Q}_{p} .
$$




\section{$9 \quad M_{1} Q(\ell)$ : Eisenstein series and the $\alpha$-family}

Let $p \geq 5$ and assume that $\ell$ is a topological generator of $\mathbb{Z}_{p}^{\times}$. In this section we will compute

$$
H^{0}\left(C^{\bullet}(\ell) / p^{\infty}\right),
$$

the 0 th cohomology of the cochain complex associated to the cosimplicial abelian group $C^{\bullet}(\ell)$ tensored with the group $\mathbb{Z} / p^{\infty}$. These computations will allow us to determine part of the 1 -line of the chromatic spectral sequence for $Q(\ell)$.

We have

$$
H^{0}\left(C^{\bullet}(\ell) / p^{\infty}\right)=\underset{j}{\lim } H^{0}\left(C^{\bullet}(\ell) / p^{j}\right),
$$

so it suffices to compute the latter. Our explicit determination of the first differential in $C^{\bullet}(\ell)$ implies that

$$
\begin{aligned}
\mathcal{A}_{t / j} & :=H^{0}\left(C^{\bullet}(\ell) / p^{j}\right)_{2 t} \\
& =\left\{f \in\left(M_{t}\right)_{\mathbb{Z} / p^{j}}^{0}: \begin{array}{ll}
\text { (i) } & \left(\ell^{t}-1\right) f(q) \equiv 0 \bmod p^{j}, \\
& \text { (ii) } \ell^{t} f\left(q^{\ell}\right)-f(q) \equiv 0 \bmod p^{j} .
\end{array}\right\}
\end{aligned}
$$

Lemma 9.1 A modular form $f \in M_{t}^{0}$ represents an element of the group $\mathcal{A}_{t / j}$ if and only if

(1) $p^{i} f \equiv 0 \bmod p^{j}$ for $t=(p-1) p^{i-1} s$, and $(s, p)=1$,

(2) $f(q) \equiv a \bmod p^{j}$ for $a \in \mathbb{Z} / p^{j}$.

Proof Since $\ell$ was assumed to be a topological generator of $\mathbb{Z}_{p}$,

$$
v_{p}\left(\ell^{t}-1\right)=i
$$

for $t=(p-1) p^{i-1} s$, with $(s, p)=1$. Condition (i) of (9-2) states that

$$
\left(\ell^{t}-1\right) f \equiv 0 \bmod p^{j} .
$$

This proves (1).

Because $\ell^{t} f(q) \equiv f(q)$ mod $p^{j}$, we deduce that condition (ii) of (9-2) may be rewritten as

$$
f\left(q^{\ell}\right) \equiv f(q) \bmod p^{j} .
$$

But, writing

$$
f(q)=\sum_{n} a_{n} q^{n}
$$


for $a_{n} \in \mathbb{Z} / p^{j}$, we see that

$$
a_{n} \equiv\left\{\begin{array}{lll}
0, & n \equiv 0 & \bmod \ell, \\
a_{n / \ell}, & n \neq 0 & \bmod \ell .
\end{array}\right.
$$

Therefore, we inductively deduce that $a_{n} \equiv 0 \bmod p^{j}$ unless $n=0$.

Let $E_{k} \in M_{k}$ denote the weight $k$ normalized Eisenstein series (for $k \geq 4$ even), with $q$-expansion

$$
E_{k}(q)=1-\frac{2 k}{B_{k}} \sum_{i=1}^{\infty} \sigma_{k-1}(i) q^{i} \in \mathbb{Q}[[q]],
$$

where

$$
\sigma_{k}(i):=\sum_{d \mid i} d^{k}
$$

The following lemma follows immediately from the Clausen-von Staudt Theorem on denominators of Bernoulli numbers.

Lemma 9.2 If $p-1$ divides $k$, the $q$-expansion of $E_{k}$ is $p$-integral. For $k \equiv 0$ $\bmod (p-1) p^{j-1}$ we have

$$
E_{k}(q) \equiv 1 \bmod p^{j}
$$

Lemma 9.3 For each even weight $k \geq 4$ there exists a modular form

$$
e_{k} \in\left(M_{k}\right)_{\mathbb{Z}_{(p)}}^{0}
$$

such that

(1) if $k \equiv 0 \bmod p-1$, we have

$$
e_{k}=E_{k}
$$

(2) the $q$-expansion of $e_{k}$ satisfies

$$
e_{k}(q)=1+\text { higher terms, }
$$

(3) if $k_{1} \equiv k_{2} \bmod (p-1) p^{j-1}$, then

$$
e_{k_{1}}(q) \equiv e_{k_{2}}(q) \bmod p^{j}
$$


Proof Observe that for any even $k \geq 4$, there exist modular forms $e_{k}$ satisfying condition (2) (one can simply take $e_{k}=E_{4}^{i} E_{6}^{j}$ for appropriate $i$ and $j$ ). Fix such choices of $e_{k}$ for even $k$ satisfying $4 \leq k<p-1$ and $k=p+1$. Also set $e_{0}=1$. For even $k \geq p-1$ satisfying $k \neq p+1$ set

$$
e_{k}=e_{k-(p-1) t} E_{(p-1) t}
$$

for $t$ chosed such that

$$
0 \leq k-(p-1) t<p-1 \text { or } k-(p-1) t=p+1 .
$$

Then condition (1) is obviously satisfied, and condition (3) is satisfied by Lemma 9.2 .

The following lemma provides a convenient basis for $p$-integral modular forms which we shall make frequent use of.

Lemma 9.4 The forms

$$
\left\{\Delta^{k} e_{t-12 k}: k \in \mathbb{Z}, t-12 k \geq 4 \text { and even }\right\},
$$

together with

$$
\Delta^{k} \text { if } t=12 k
$$

form an integral basis of $M_{t}^{0}$.

\section{Proof Since}

$$
\Delta(q)=q+\cdots
$$

we have

$$
\Delta^{k}(q) e_{t-12 k}=q^{k}+\cdots .
$$

This establishes linear independence. We may deduce that these forms span $M_{t}^{0}$ by the explicit calculation

$$
M_{*}=\mathbb{Z}\left[E_{4}, E_{6}, \Delta^{-1}\right] /\left(\Delta=\frac{E_{4}^{3}-E_{6}^{2}}{1728}\right) .
$$

Proposition 9.5 The groups $\mathcal{A}_{t / \infty}=\operatorname{colim}_{j} \mathcal{A}_{t / j}$ are given by

$$
\mathcal{A}_{t / \infty}=\mathbb{Z} / p^{j}\left\{E_{t} / p^{j}\right\}
$$

for $t=(p-1) p^{j-1} s$, where $(s, p)=1$ and $t \geq 4$, and

$$
\mathcal{A}_{0 / \infty}=\mathbb{Z} / p^{\infty} \text {. }
$$

(Here, the element $E_{t} / p^{j}$ is the image of the element $E_{t} \in \mathcal{A}_{t / j}$.) 
Proof This follows immediately from Lemma 9.1 and Lemma 9.4, provided we can show that $E_{t}$ lies in $\mathcal{A}_{t / j}$. This again follows from criterion (2) of Lemma 9.1: by Lemma 9.2

$$
E_{t}(q) \equiv 1 \quad \bmod p^{j} .
$$

We obtain the zero-line of spectral sequence (7-2) as a corollary.

Corollary 9.6 We have

$$
H^{0}\left(C^{\bullet}(\ell) / p^{\infty}\left[v_{1}^{-1}\right]\right)_{2 t} \cong \begin{cases}\mathbb{Z} / p^{j}, & t=(p-1) p^{j-1} s \text { and }(s, p)=1, \\ 0, & t \neq 0 \bmod (p-1) .\end{cases}
$$

Combining this with Corollary 7.3 and Proposition 8.1, we find the following.

Corollary 9.7 We have

$$
\pi_{t} M_{1} Q(\ell) \cong \begin{cases}\mathbb{Z} / p^{j}, & t=2(p-1) p^{j-1} s \text { and }(s, p)=1, \\ 0, & t \not \equiv 0,-1,-2 \bmod 2(p-1) .\end{cases}
$$

\section{$10 \operatorname{Mod} p^{j}$ congruences}

Let $p \geq 5$. The congruence

$$
E_{p-1}(q) \equiv 1 \quad \bmod p
$$

implies the congruence

$$
E_{p-1}^{p^{j-1}}(q) \equiv 1 \quad \bmod p^{j}
$$

It follows that multiplication by $E_{p-1}^{p^{j-1}}$ induces an injection

$$
\cdot E_{p-1}^{p^{j-1}}: M_{t}\left(\Gamma_{0}(N)\right)_{\mathbb{Z} / p^{j}} \hookrightarrow M_{t+(p-1) p^{j-1}}\left(\Gamma_{0}(N)\right)_{\mathbb{Z} / p^{j}} .
$$

(Here we regard $E_{p-1}$ as a modular form for $\Gamma_{0}(N)$.) The image of this inclusion is characterized by the following theorem of Serre [12, Corollary 4.4.2].

Theorem 10.1 (Serre) Let $f_{i}$ be an elements of $M_{k_{i}}\left(\Gamma_{0}(N)\right)_{\mathbb{Z} / p^{j}}$ for $i=1,2$ and $k_{1}<k_{2}$. Then

$$
f_{1}(q)=f_{2}(q) \in \mathbb{Z} / p^{j}[[q]]
$$

if and only if

(1) $k_{1} \equiv k_{2} \bmod (p-1) p^{j-1}$, and

(2) $f_{2}=E_{p-1}^{\frac{k_{2}-k_{1}}{p-1}} f_{1}$. 


\section{$11 M_{2} Q(\ell)$ : The $\beta$-family congruences}

Let $p \geq 5$, and let $\ell$ be a topological generator of $\mathbb{Z}_{p}^{\times}$. In this section we prove Theorem 1.2 and Theorem 1.3. The key observation is the following.

Lemma 11.1 The inclusion $\Gamma_{\ell} \hookrightarrow \mathbb{S}_{2}$ induces an isomorphism

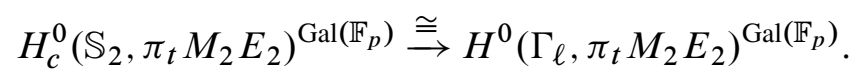

Proof By Theorem 4.1, the group $\Gamma_{\ell}$ is dense in $\mathbb{S}_{2}$. Since $\mathbb{S}_{2}$ acts continuously on $\pi_{t} M_{2} E_{2}$, the invariants of $\mathbb{S}_{2}$ are the same as the invariants of $\Gamma_{\ell}$.

Combined with Corollary 7.7, we have an isomorphism

$$
H^{0}\left(C(\ell)^{\bullet} /\left(p^{\infty}, v_{1}^{\infty}\right)\right)_{t} \cong H_{c}^{0}\left(\mathbb{S}_{2}, \pi_{t} M_{2} E_{2}\right)^{\mathrm{Gal}\left(\mathbb{F}_{p}\right)} .
$$

The right-hand side has been computed by Miller-Ravenel-Wilson [16].

Theorem 11.2 (Miller-Ravenel-Wilson) The groups $H_{c}^{0}\left(\mathbb{S}_{2}, \pi_{*} M_{2} E_{2}\right)^{\mathrm{Gal}\left(\mathbb{F}_{p}\right)}$ are generated by elements

$$
\beta_{i / j, k} \in H_{c}^{0}\left(\mathbb{S}_{2}, \pi_{2 i\left(p^{2}-1\right)-2 j(p-1)} M_{2} E_{2}\right)^{\mathrm{Gal}\left(\mathbb{F}_{p}\right)}
$$

which generate cyclic summands of order $p^{k}$. Here, for $i=s p^{n}$ with $(s, p)=1$, the indices $j$ and $k$ are taken subject to

(1) $p^{k-1} \mid j$,

(2) $j \leq p^{n-k+1}+p^{n-k}-1$,

(3) either $j>p^{n-k}+p^{n-k-1}-1$ or $p^{k} \chi j$.

We now compute

$$
H^{0}\left(C^{\bullet}(\ell) /\left(p^{\infty}, v_{1}^{\infty}\right)\right)_{*}
$$

in terms of modular forms.

We have

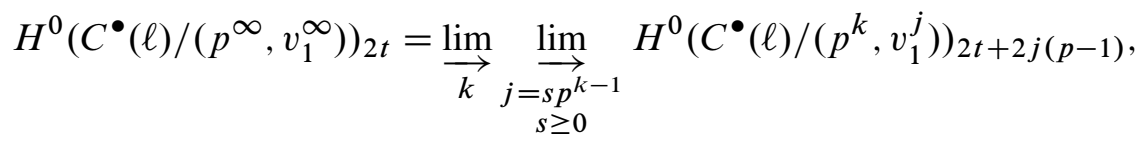


so it suffices to compute the latter. Proposition 6.1, Lemma 7.2, and Theorem 10.1 imply that, for $j \equiv 0 \bmod p^{k-1}$, we have:

$$
\begin{aligned}
& \mathcal{B}_{t / j, k}:=H^{0}\left(C^{\bullet}(\ell) /\left(p^{k}, v_{1}^{j}\right)\right)_{2 t+2 j(p-1)} \\
& =\operatorname{ker}\left(\begin{array}{ll}
\frac{M_{t+j(p-1)}^{0}}{\left(p^{k}, E_{p-1}^{j}\right)} \stackrel{d_{0}-d_{1}}{\longrightarrow} & \frac{M_{t+j(p-1)}^{0}}{\left(p^{k}, E_{p-1}^{j}\right)} \\
\frac{M_{t+j(p-1)}\left(\Gamma_{0}(\ell)\right)^{0}}{\left(p^{k}, E_{p-1}^{j}\right)}
\end{array}\right) \\
& =\operatorname{ker}\left(\begin{array}{cc}
\left(M_{t+j(p-1)}\right)_{\mathbb{Z} / p^{k}}^{0} \stackrel{\frac{\left(M_{t+j(p-1)}\right)_{\mathbb{Z} / p^{k}}^{0}}{\left(M_{t}\right)_{\mathbb{Z} / p^{k}}^{0}}}{\left(M_{t}\right)_{\mathbb{Z} / p^{k}}^{0}} & \bigoplus \\
\frac{M_{t+j(p-1)}\left(\Gamma_{0}(\ell)\right)_{\mathbb{Z} / p^{k}}^{0}}{M_{t}\left(\Gamma_{0}(\ell)\right)_{\mathbb{Z} / p^{k}}^{0}}
\end{array}\right)
\end{aligned}
$$

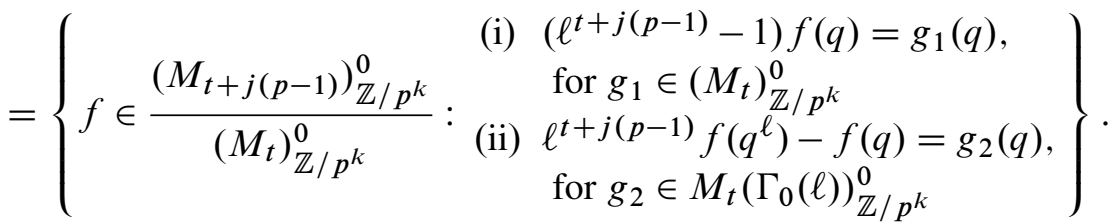

Here, we are regarding the space of mod $p^{k}$ modular forms of weight $t$ as being embedded in the space of mod $p^{k}$ modular forms of weight $t+j(p-1)$ through the inclusion induced by multiplication by $E_{p-1}^{j}$ using Theorem 10.1.

For a finitely generated abelian $p$-group $A$, we shall say that $a \in A$ is an additive generator of order $p^{k}$ if $a$ generates a cyclic subgroup of $A$ of order $p^{k}$.

Theorem 11.3 There is a one-to-one correspondence between the additive generators of order $p^{k}$ in

$$
H^{0}\left(C(\ell)^{\bullet} /\left(p^{\infty}, v_{1}^{\infty}\right)\right)_{2 t}
$$

and the modular forms $f \in M_{t+j(p-1)}^{0}$ for $j \equiv 0 \bmod p^{k-1}$ satisfying the following.

(1) We have $t \equiv 0 \bmod (p-1) p^{k-1}$.

(2) The $q$-expansion $f(q)$ is not congruent to $0 \bmod p$.

(3) We have $\operatorname{ord}_{q} f(q)>\frac{t}{12}$ or $\operatorname{ord}_{q} f(q)=\frac{t-2}{12}$.

(4) There does not exist a form $f^{\prime} \in M_{t^{\prime}}^{0}$ such that $f^{\prime}(q) \equiv f(q) \bmod p^{k}$ for $t^{\prime}<t+j(p-1)$. 
$(5)_{\ell}$ There exists a form

$$
g \in M_{t}\left(\Gamma_{0}(\ell)\right)^{0}
$$

satisfying

$$
f\left(q^{\ell}\right)-f(q) \equiv g(q) \bmod p^{k}
$$

We will need to make use of the following lemma.

Lemma 11.4 There exist homomorphisms

$$
r_{m}: M_{t+m}^{0} \rightarrow M_{t}^{0}
$$

such that, if $j \equiv 0 \bmod p^{k-1}$, the short exact sequences

$$
0 \rightarrow\left(M_{t}\right)_{\mathbb{Z} / p^{k}}^{0} \stackrel{\cdot E_{p-1}^{j}}{\longrightarrow}\left(M_{t+j(p-1)}\right)_{\mathbb{Z} / p^{k}}^{0} \rightarrow \frac{\left(M_{t+j(p-1)}\right)_{\mathbb{Z} / p^{k}}^{0}}{\left(M_{t}\right)_{\mathbb{Z} / p^{k}}^{0}} \rightarrow 0
$$

are split by the mod $p^{k}$ reduction of $r_{j(p-1)}$.

Proof Using the basis of Lemma 9.4 we define explicit splitting morphisms

$$
r_{m}: M_{t+m} \rightarrow M_{t}
$$

whose effect on basis vectors is given by

$$
r_{m}\left(\Delta^{n} e_{t+m-12 n}\right)= \begin{cases}\Delta^{n} e_{t-12 n}, & t-12 n=0, \text { or } t-12 n=2 i \text { for } i \geq 2, \\ 0, & \text { otherwise. }\end{cases}
$$

We just need to verify that $r_{j(p-1)}$ reduces to give the appropriate splittings. By Condition (3) of Lemma 9.3, and (10-1), we have

$$
e_{t}(q) E_{p-1}^{j}(q) \equiv e_{t}(q) \equiv e_{t+j(p-1)}(q) \bmod p^{k} .
$$

We therefore compute

$$
\begin{aligned}
r_{j(p-1)}\left(\Delta^{n} e_{t-12 n} E_{p-1}^{j}\right) & \equiv r_{j(p-1)}\left(\Delta^{n} e_{t+j(p-1)-12 n}\right) \bmod p^{k} \\
& \equiv \Delta^{n} e_{t-12 n} \bmod p^{k} .
\end{aligned}
$$

The splittings of Lemma 11.4 induce splitting homomorphisms which give short exact sequences

$$
0 \leftarrow\left(M_{t}\right)_{\mathbb{Z} / p^{k}}^{0} \stackrel{r_{j, k}}{\longleftarrow}\left(M_{t+j(p-1)}\right)_{\mathbb{Z} / p^{k}}^{0} \stackrel{\iota_{j, k}}{\longleftarrow} \frac{\left(M_{t+j(p-1)}\right)_{\mathbb{Z} / p^{k}}^{0}}{\left(M_{t}\right)_{\mathbb{Z} / p^{k}}^{0}} \leftarrow 0 .
$$

These short exact sequences are compatible as $k$ and $j$ vary. 
Lemma 11.5 For $t$ even, the image of the homomorphism $\iota_{j, k}$ is given by

$$
\left\{f \in\left(M_{t+j(p-1)}\right)_{\mathbb{Z} / p^{k}}^{0}: \operatorname{ord}_{q} f(q)>\frac{t}{12} \text { or } \operatorname{ord}_{q} f(q)=\frac{t-2}{12}\right\} .
$$

Proof A basis of $\left(M_{t}\right)_{\mathbb{Z} / p^{k}}^{0}$ is given by

$$
\left\{\Delta^{n} e_{t-12 n}: n \leq \frac{t}{12}, n \neq \frac{t-2}{12}\right\} .
$$

The image of this basis under $\iota_{j, k}$ is spanned by

$$
\left\{\Delta^{n} e_{t-12 n+j(p-1)}: n \leq \frac{t}{12}, n \neq \frac{t-2}{12}\right\} \text {. }
$$

Since

$$
\Delta^{n} e_{t-12 n+j(p-1)}=q^{n}+\cdots
$$

we deduce the result.

Proof of Theorem 11.3 Suppose that $b^{\prime} \in \mathcal{B}_{t / j, k^{\prime}}$ is an additive generator of order $p^{k}$. Let $f^{\prime}$ be the lift

$$
f^{\prime}:=\iota_{j, k^{\prime}}\left(b^{\prime}\right) \in\left(M_{t+j(p-1)}\right)_{\mathbb{Z} / p^{k^{\prime}}}^{0}
$$

Since $b^{\prime}$ is assumed to be an additive generator of order $p^{k}$ and $\iota_{j, k^{\prime}}$ is injective, we deduce that $f^{\prime}$ is a modular form in $\left(M_{t+j(p-1)}\right)_{\mathbb{Z} / p^{k^{\prime}}}$ of exact order $p^{k}$. Hence $f^{\prime}=p^{k^{\prime}-k} f$ for some modular form $f \in\left(M_{t+j(p-1)}\right)_{\mathbb{Z} / p^{k}}^{0}$. It is simple to check that the image

$$
b \in \frac{\left(M_{t+j(p-1)}\right)_{\mathbb{Z} / p^{k}}^{0}}{\left(M_{t}\right)_{\mathbb{Z} / p^{k}}^{0}}
$$

represents an element of $\mathcal{B}_{t / j, k}$.

It follows that the additive generators of order $p^{k}$ in

$$
H^{0}\left(C(\ell)^{\bullet} /\left(p^{\infty}, v_{1}^{\infty}\right)\right)_{t}=\operatorname{colim}_{k^{\prime}} \operatorname{colim}_{\substack{j=s k^{\prime}-1 \\ s \geq 1}} \mathcal{B}_{t / j, k^{\prime}}
$$

exactly correspond to the additive generators of order $p^{k}$ in $\mathcal{B}_{t / j, k}$ which are not in the image of the inclusion

$$
\cdot E_{p-1}^{p^{k-1}}: \mathcal{B}_{t / j-p^{k-1, k}} \hookrightarrow \mathcal{B}_{t / j, k} \cdot
$$

Suppose that $b$ is such an additive generator. Let $f$ be the lift

$$
f:=\iota_{j, k}(b) \in\left(M_{t+j(p-1)}\right)_{\mathbb{Z} / p^{k}}^{0} .
$$


Then by Lemma 11.5 the lift $f$ satisfies

$$
\operatorname{ord}_{q} f(q)>\frac{t}{12} \text { or } \operatorname{ord}_{q} f(q)=\frac{t-2}{12} .
$$

From the definition of $\mathcal{B}_{t / j, k}$ we have

(i) $\left(\ell^{t+j(p-1)}-1\right) f(q)=g_{1}(q)$,

$$
\text { for } g_{1} \in\left(M_{t}\right)_{\mathbb{Z} / p^{k}}^{0}
$$

(ii) $\ell^{t+j(p-1)} f\left(q^{\ell}\right)-f(q)=g_{2}(q)$,

$$
\text { for } g_{2} \in M_{t}\left(\Gamma_{0}(\ell)\right)_{\mathbb{Z} / p^{k}}^{0} \text {. }
$$

Since $j \equiv 0 \bmod p^{k-1}$ we deduce that

$$
\ell^{t+j(p-1)} \equiv \ell^{t} .
$$

Let $v=v_{p}\left(\ell^{t}-1\right)$. Condition (i) above implies that

$$
f(q) \equiv \frac{g_{1}(q)}{\ell^{t}-1} \quad \bmod p^{k-v} .
$$

But, if $b^{\prime \prime} \in \mathcal{B}_{t / j, k-v}$ is the image of the mod $p^{k-v}$ reduction of $b$, then

$$
f(q) \equiv \iota_{j, k-v}\left(b^{\prime \prime}\right) \quad \bmod p^{k-v}
$$

and thus, by the exactness of (11-1), we have

$$
r_{j, k-v}(f)=g_{1}=0 .
$$

Thus we actually have

$$
\left(\ell^{t}-1\right) f(q) \equiv 0 \quad \bmod p^{k} .
$$

Since $f(q)$ has order $p^{k}$, we deduce that

$$
\ell^{t} \equiv 1 \bmod p^{k}
$$

Since $\ell$ is a topological generator of $\mathbb{Z}_{p}^{\times}$, we deduce that

$$
t \equiv 0 \bmod (p-1) p^{k-1} .
$$

Thus condition (ii) may be rewritten as

$$
f\left(q^{\ell}\right)-f(q)=g_{2}(q) \text { for } g_{2} \in M_{t}\left(\Gamma_{0}(\ell)\right)_{\mathbb{Z} / p^{k}}^{0} .
$$

We have therefore verified conditions (1)-(5) of Theorem 11.3. 
For the converse direction, suppose $f \in\left(M_{t+j(p-1)}\right)_{\mathbb{Z} / p^{k}}$ satisfies conditions (1)-(5) of Theorem 11.3. Then by Lemma 11.5, $f$ is in the image of $\iota_{j, k}$. Consider the image

$$
b=[f] \in \frac{\left(M_{t+j(p-1)}\right)_{\mathbb{Z} / p^{k}}^{0}}{\left(M_{t}\right)_{\mathbb{Z} / p^{k}}^{0}}
$$

of $f$ in the quotient. Observe that by (2), the element $b$ has order $p^{k}$. We just need to verify that it is an element of $\mathcal{B}_{t / j, k}$, which amounts to seeing that $f$ satisfies conditions (i) and (ii) above. But condition (1) implies that

$$
\ell^{t} \equiv \ell^{t+j(p-1)} \equiv 1 \quad \bmod p^{k} .
$$

This immediately implies that $f$ satisfies condition (i). Condition (ii) then follows from condition (5).

Observe that if $S$ is a set of primes which contains $\ell$ and does not contain $p$, then we have

$$
\Gamma_{\ell} \subseteq \Gamma_{S} \subset \mathbb{S}_{2} .
$$

Since $\Gamma_{\ell}$ is dense in $\mathbb{S}_{2}$, the subgroup $\Gamma_{S}$ is dense in $\mathbb{S}_{2}$. We therefore deduce the following lemma.

Lemma 11.6 For a set of primes $S$ not containing $p$ and containing $\ell$, there is an isomorphism

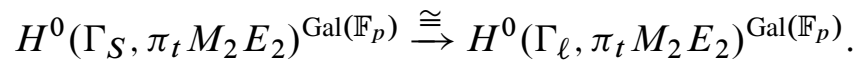

In particular, letting $\ell^{\prime}$ be a prime in $S$, we have a zig-zag

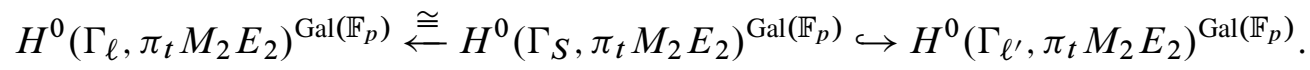

If $\ell^{\prime}$ also generates $\mathbb{Z}_{p}^{\times}$, then the inclusion is an isomorphism. Corollary 7.7 allows us to deduce the following.

Corollary 11.7 For any prime $\ell^{\prime} \neq \ell$ There is an inclusion

$$
H^{0}\left(C(\ell)^{\bullet} /\left(p^{\infty}, v_{1}^{\infty}\right)\right)_{t} \hookrightarrow H^{0}\left(C\left(\ell^{\prime}\right)^{\bullet} /\left(p^{\infty}, v_{1}^{\infty}\right)\right)_{t} .
$$

If $f$ satisfies Conditions (1)-(4) and (5) $\ell$ of Theorem 11.3, then it satisfies condition $(5)_{\ell^{\prime}}$

We finish this section by observing that the results of this section combine to give proofs of some of the theorems stated in Section 1. 
Proofs of Theorem 1.2, Theorem 1.3 and Theorem 1.4 Corollary 11.7 implies Theorem 1.4. The element

$$
\beta_{i / j, k} \in H^{0}\left(\mathbb{S}_{2}, \pi_{2 i\left(p^{2}-1\right)-2 j(p-1)} M_{2} E_{2}\right)^{\mathrm{Gal}\left(\mathbb{F}_{p}\right)}
$$

detects a corresponding Greek letter element

$$
\beta_{i / j, k} \in \operatorname{Ext}_{B P_{*} B P}^{2, *}\left(B P_{*}, B P_{*}\right)
$$

in the chromatic spectral sequence if $i>0$ and $i \neq p^{n}$ [16] (if $i=p^{n}$, then $j$ must be greater than or equal to $p^{n}$ ). Thus Theorem 1.2 and Theorem 1.3 follow from Theorem 11.3. Note that the modular forms $f=f_{i / j, k}$ of Theorem 1.2 and Theorem 1.3 are taken to be holomorphic at the cusps, whereas in Theorem 11.3, they are merely assumed to be meromorphic at the cusps. This discrepancy is resolved by noting that if $i, j, k$ are chosen such that $\beta_{i / j, k}$ exists in $\operatorname{Ext}_{B P_{*} B P}\left(B P_{*}, B P_{*}\right)$, then

$$
t=i\left(p^{2}-1\right)-j(p-1) \geq 0 .
$$

Therefore, condition (2) of Theorem 11.3 guarantees that the modular forms in question are holomorphic at the cusps.

\section{Greek letter elements in the Hurewicz image of $Q(\ell)$}

Since the cosimplicial spectrum $Q(\ell)^{\bullet}$ is a cosimplicial object in the category of $E_{\infty}$-ring spectra, the equivalence $Q(\ell) \simeq$ Tot $Q(\ell)^{\bullet}(5-4)$ allows us to regard $Q(\ell)$ as an $E_{\infty}$-ring spectrum. In particular, it possesses a unit map

$$
S \rightarrow Q(\ell)
$$

which, by Lemma 7.1, localizes to give a map

$$
S_{E(2)} \rightarrow Q(\ell) .
$$

In this section we prove the following.

Theorem 12.1 The images of the elements $\alpha_{i / j}$ and the elements $\beta_{i / j, k}$ under the homomorphism

$$
\pi_{*} S_{E(2)} \rightarrow \pi_{*} Q(\ell)
$$

are non-trivial.

We first will need a lemma. 


\section{Lemma 12.2 The map}

$$
\pi_{t} M_{1} S \rightarrow \pi_{t} M_{1} Q(\ell)
$$

is an isomorphism for $t \equiv 0 \bmod 4$.

Proof Let $C$ be an ordinary elliptic curve over $\overline{\mathbb{F}}_{p}$, so that there is an isomorphism of formal groups

$$
C^{\wedge} \cong \widehat{\mathbb{G}}_{m} \text {. }
$$

Let $U$ be the formal neighborhood of the associated point of $\mathcal{M}\left(K_{0}^{p}\right)$, which carries a universal deformation $\widetilde{C} / U$ of $C$. Let

$$
E=\mathcal{E}_{K_{0}^{p}}(U)
$$

be the sections of the sheaf $\mathcal{E}$ over $U$. By Serre-Tate theory, and the deformation theory of $p$-divisible groups $[6$, Section 7.1$]$, we deduce that

$$
U \cong \operatorname{Spf}\left(W\left(\overline{\mathbb{F}}_{p}\right)[[x]]\right)
$$

and therefore that $E$ is an even periodic ring spectrum with $\pi_{0}(E) \cong W\left(\overline{\mathbb{F}}_{p}\right)[[x]]$, with associated formal group given by $\widetilde{C}^{\wedge}$. The cofiber

$$
E \stackrel{\cdot x}{\rightarrow} E \rightarrow E / x
$$

is an even periodic ring spectrum (Elmendorf-Kriz-Mandell-May [8]). The restriction $\widetilde{C}_{\text {can }}$ of the deformation $\widetilde{C}$ to $\pi_{0}(E / x) \cong W\left(\overline{\mathbb{F}}_{p}\right)$ is the canonical deformation of $C$ (the deformation whose $p$-divisible group splits). The formal group $\widetilde{C}_{\text {can }}^{\wedge}$ is therefore a universal deformation of $\mathbb{G}_{m} / \overline{\mathbb{F}}_{p}$, and we conclude that there is an isomorphism

$$
\widetilde{C}_{\text {can }}^{\wedge} \cong \widehat{\mathbb{G}}_{m}
$$

between the formal group for $E / x$ and the multiplicative formal group. In particular, this implies that there is an equivalence of ring spectra

$$
K_{p} \otimes_{\mathbb{Z}_{p}} W\left(\overline{\mathbb{F}}_{p}\right) \cong E / x,
$$

where $K_{p}$ is the $p$-adic $K$-theory spectrum. Now, the unit map $S \rightarrow K_{p}$ induces an inclusion

$$
\pi_{2 t} M_{1} S \hookrightarrow \pi_{2 t} M_{1} K_{p}
$$

(it gives the Adams $e$-invariant). Therefore the unit map for $E / x$ induces an inclusion

$$
\pi_{2 t} M_{1} S \hookrightarrow \pi_{2 t} M_{1}(E / x) .
$$

However, the unit for $E / x$ is homotopic to the composite

$$
S \rightarrow Q(\ell) \simeq \operatorname{Tot} Q(\ell)^{\bullet} \rightarrow Q(\ell)^{0}=\mathrm{TMF}_{p} \rightarrow E \rightarrow E / x
$$


because all of the maps in the composite are maps of ring spectra. We deduce that the maps

$$
\pi_{t} M_{1} S \rightarrow \pi_{t} M_{1} Q(\ell)
$$

are injective for $t \equiv 0 \bmod 2$. By Corollary 9.7, these (finite) groups are abstractly isomorphic for $t \equiv 0 \bmod 4$ and $t \neq 0$. The result for $t \neq 0$ therefore is proven. The cases of $t=0$ follows immediately from the fact that the map $\pi_{0} S \rightarrow \pi_{0} Q(\ell) \cong \mathbb{Z}_{p}$ is a map of rings.

Proof of Theorem 12.1 Consider the map of chromatic spectral sequences:

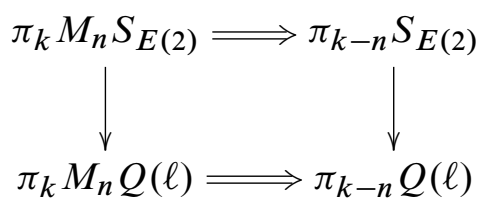

The elements $\alpha_{i / j} \in \pi_{2 i(p-1)}\left(M_{1} S\right)$ are known to be permanent cycles for $i>0$, and therefore map to permanent cycles in the chromatic spectral sequence for $Q(\ell)$. By Lemma 12.2, the images of $\alpha_{i / j}$ in $\pi_{t} M_{1} S$ are nontrivial, and generate these groups for $t \equiv 0 \bmod 4$. Since, by Corollary 8.4, $\pi_{t} M_{0} Q(\ell)$ is zero for $t \neq 0,-1,-2$, there are no non-trivial differentials

$$
d_{1}: \pi_{t} M_{0} Q(\ell) \rightarrow \pi_{t} M_{1} Q(\ell)
$$

for $t>0$. We deduce that the images of the elements $\alpha_{i / j}$ in the chromatic spectral sequence for $Q(\ell)$ are non-trivial permanent cycles, and hence witness the non-triviality of the images of the elements $\alpha_{i / j}$ in $\pi_{*} Q(\ell)$. As a side-effect, we have also determined that the groups $\pi_{t} M_{1} Q(\ell)$ are generated by permanent cycles for $t \equiv 0 \bmod 4$. That, combined with the fact that $\pi_{t} M_{0} Q(\ell)$ is zero for $t$ positive, allows us to deduce that there are no non-trivial differentials killing elements of $\pi_{t} M_{2} Q(\ell)$ for $t \equiv 0 \bmod 4$. To complete the proof of the theorem, it suffices to show that the images of the elements $\beta_{i / j, k}$ are non-trivial under the homomorphism

$$
\pi_{t} M_{2} S \rightarrow \pi_{t} M_{2} Q(\ell)
$$

where $t=2 i\left(p^{2}-1\right)-2 j(p-1)$. But, for such $t$, the map (12-1) is given by the composite of isomorphisms

$$
\begin{aligned}
\pi_{t} M_{2} S & \cong H_{c}^{0}\left(\mathbb{S}_{2}, \pi_{t} M_{2} E_{2}\right)^{\mathrm{Gal}\left(\mathbb{F}_{p}\right)} \\
& \cong H^{0}\left(\Gamma_{\ell}, \pi_{t} M_{2} E_{2}\right)^{\mathrm{Gal}\left(\mathbb{F}_{p}\right)} \\
& \cong \pi_{t} M_{2} Q(\ell)
\end{aligned}
$$

given by Corollary 2.2, Lemma 11.1, and Lemma 7.6. 


\section{References}

[1] A Baker, Hecke operations and the Adams $E_{2}$-term based on elliptic cohomology, Canad. Math. Bull. 42 (1999) 129-138 MR1692001

[2] M Behrens, A modular description of the $K(2)$-local sphere at the prime 3, Topology 45 (2006) 343-402 MR2193339

[3] M Behrens, Buildings, elliptic curves, and the K(2)-local sphere, Amer. J. Math. 129 (2007) 1513-1563 MR2369888

[4] M Behrens, Some root invariants at the prime 2, Geom. Topol. Monogr. 10 (2007) $1-40$

[5] M Behrens, G Laures, $\beta$-family congruences and the $f$-invariant, preprint

[6] M Behrens, T Lawson, Topological automorphic forms, to appear in memoirs of the AMS

[7] M Behrens, T Lawson, Isogenies of elliptic curves and the Morava stabilizer group, J. Pure Appl. Algebra 207 (2006) 37-49 MR2244259

[8] A D Elmendorf, I Kriz, M A Mandell, J P May, Rings, modules, and algebras in stable homotopy theory, Mathematical Surveys and Monographs 47, American Mathematical Society, Providence, RI (1997) MR1417719 With an appendix by M Cole

[9] H Hida, p-adic automorphic forms on Shimura varieties, Springer Monographs in Mathematics, Springer, New York (2004) MR2055355

[10] M J Hopkins, J H Smith, Nilpotence and stable homotopy theory. II, Ann. of Math. (2) 148 (1998) 1-49 MR1652975

[11] J Hornbostel, N Naumann, Beta-elements and divided congruences, Amer. J. Math. 129 (2007) 1377-1402 MR2354323

[12] N M Katz, p-adic properties of modular schemes and modular forms, from: "Modular functions of one variable, III (Proc. Internat. Summer School, Univ. Antwerp, Antwerp, 1972)”, Springer, Berlin (1973) 69-190. Lecture Notes in Mathematics, Vol. 350 MR0447119

[13] S S Kudla, From modular forms to automorphic representations, from: "An introduction to the Langlands program (Jerusalem, 2001)”, Birkhäuser, Boston (2003) 133-151 MR1990378

[14] G Laures, The Topological q-Expansion Principle, PhD thesis, MIT (1996)

[15] G Laures, The topological q-expansion principle, Topology 38 (1999) 387-425 MR1660325

[16] H R Miller, D C Ravenel, W S Wilson, Periodic phenomena in the Adams-Novikov spectral sequence, Ann. Math. (2) 106 (1977) 469-516 MR0458423

[17] J Morava, Noetherian localisations of categories of cobordism comodules, Ann. of Math. (2) 121 (1985) 1-39 MR782555 
[18] D C Ravenel, Localization with respect to certain periodic homology theories, Amer. J. Math. 106 (1984) 351-414 MR737778

[19] J H Silverman, The arithmetic of elliptic curves, Graduate Texts in Mathematics 106, Springer, New York (1986) MR817210

[20] W C Waterhouse, Abelian varieties over finite fields, Ann. Sci. École Norm. Sup. (4) 2 (1969) 521-560 MR0265369

MIT Department of Mathematics 2-273, 77 Massachusetts Ave Cambridge, MA 02140, USA

mbehrens@math .mit .edu

http://www-math.mit.edu/ mbehrens

Proposed: Paul Goerss

Received: 3 May 2008

Seconded: Bill Dwyer, Haynes Miller

Revised: 13 October 2008 